\title{
Interim Performance Evaluation in Contract Design
}

\author{
Bin R. Chen and Y. Stephen Chiu*
}

November 9, 2011

\begin{abstract}
In this paper we study a principal-agent problem with two-stage complementary efforts, as well as limited liability on the agent's side. An interim-performance-evaluation (IPE) allows the principal to learn of the degree of success of the first stage investment. We find conditions under which such an IPE is profitable even if the continuation action plan is constant regardless of the IPE outcome. The conditions are related to the degree of task complementarity, which turns out to have a close relationship between the comparison of the likelihood ratios under different IPE outcomes. We also argue that notions such as morale and confidence, which are normally outside standard economic discourse, can be used to understand our results. This thus extends the line of studies on intrinsic motivation versus extrinsic incentive pioneered by Bénabou and Tirole (2003).
\end{abstract}

$J E L:$ D82, L14, M20.

\section{Introduction}

In most real world problems, the successful production of a good normally requires sequential investments. For example, building a bridge requires planning and feasibility analysis in an early stage and building of the bridge in a later stage; for another example, the completion of a doctoral degree requires the student to take course work, to pass in a qualify exam, and to conduct research and write a thesis of a reasonable quality. In many such examples, interim performance evaluations

${ }^{*}$ We would like to thank Kalyan Chatterjee, Stephen Ching, Jacques Crémer, Jin Li, Ping Lin, Wing Suen, Zhigang Tao, Frances Xu and participants in the 2010 Econometric Society World Congress (Shanghai) for helpful discussions and suggestions. All remaining errors are ours.

${ }^{\dagger}$ Chen: School of Business, Sun Yat-sen University, Guangzhou, CHINA. Email: xiechenbin@gmail.com. Chiu (corresponding author): School of Economics and Finance, University of Hong Kong, Pokfulam Road, HONG KONG. Email:schiu@econ.hku.hk. 
(IPEs) are performed. In the case of bridge building, the feasibility analysis may turn out to suggest against building the bridge; in the case of doctoral studies, the qualify exam may turn out to advise the doctoral student be dropped from the program. The merit of such IPEs is clear when they provide new information and optimal continuation actions vary dependent on the IPE outcomes. A less clear issue is whether they are still valuable when the principal aims at the same continuation actions regardless of the IPE outcomes.

Warnings against the use of IPEs can be found among both economists and management researchers. In his recent best seller, for instance, Roberts (2004) writes that "[s]ubjective measures and milestones may provide more effective incentives for innovation than do the accounting numbers, but using them to provide very intense incentives is certainly problematic." According to two experts on project management, the risk of having IPEs is that the self-confidence of the reviewees is likely to be damaged when the IPEs turn out to be unfavorable. The reviewees are thus discouraged from working hard. "There is little doubt that these swings of mood have a destructive effect on performance...despair is even worse because the project is permeated with an attitude that says, 'Why try when we are destined to fail?'"(Meredith and Mantel 1995).

The question of the value of IPEs also appears in recent research in contract theory. Lizzeri, Meyer and Persico (2002), Fuchs (2007), and Manso (2011) contain results that support the aforementioned concern. Specifically, they show that, whenever the desired continuation action does not depend on the IPE outcomes, it is better not to reveal them to the reviewees. By not revealing, there is no need to provide differential incentives depending on the IPE outcomes (another way put, fewer incentive compatibility constraints need to be satisfied).

In this paper, we set out to study the issue through a simple two-stage principal-agent problem. The principal solicits the agent's help to produce a final good which may turn out to be a success or a failure. The success probability depends on two non-observable efforts made by the agent sequentially. The focus of analysis is where the two efforts exhibit complementarity - that is, a higher level of effort 1 makes the effort 2 more productive and vice versa. We also assume that, while both the parties are risk neutral, the agent suffers from limited liability. We think that limited liability is a reasonsable assumption in many applications. The benchmark model is a so-called traditional contract which conditions the payment to the agent on the quality of the final good.

Given this benchmark, we study a contract in which payments to the agent depend not only on the final product quality but also on the outcome of an IPE conducted at the end of the first stage. 
For simplicity, we assume that the principal can conduct an IPE costlessly, and the IPE outcome informs the principal about the success probability of the final good. A positive (negative) outcome means a high (low) probability of success and, given our assumption of effort complementarity, the increment of success probability due to the second stage effort is large (small) and this thus boosts (damages) the agent's morale. Our model allows us to understand the trade-off between the beneficial effect when his morale is boosted and the harmful effect when his morale is damaged.

The main results are the following. First, we show that, given the same continuation effort regardless of the IPE outcome, the IPE contract and the traditional contract are generally not equivalent. Second and more specifically, we find out the conditions under which the former is more profitable the latter. One necessary condition is that the use of IPE does not introduce additional binding IC constraints to the problem. One necessary condition is that the likelihood ratios of the second effort do not differ too much under different IPE outcomes, which is the case when effort complementarity is weak. On the contrary, when the complementarity is strong, or when the two likelihood ratios differ a lot, using IPE is never optimal given the same continuation effort regardless of the IPE outcome.

Third, we show that IPEs are less likely to be valuable when IPE outcomes are subjective and the principal may have an incentive to lie about the findings. Nonetheless, we find that parameter values still exist over which having IPEs is more profitable than not having them.

Fourth, we also characterize the IPE contracts when the continuation efforts depend on the IPE outcomes. Naturally this makes IPE more likely to be useful. While this is not surprising, it is still interesting comparing the difference between the optimal IPE contract given such a variable continuation action plan with the one given the same continuation action plan.

Fifth and finally, we find notions such as morale and confidence, which are normally outside standard economic discourse, to be helpful in interpreting our results. With a lower morale or selfconfidence, the agent needs to be motivated with greater-powered incentive. Bénabou and Tirole (2003) are the first to formulate self-confidence and to study the potentially harmful effect of highpowered incentives. As the principal-agent problem we study is even more standard that theirs, rhis paper echos their call for using economic modeling to analyze problems psychologists are interested in. We think that notions such as morale and self-confidence have their justifiable places in even broader economic environments.

The remainder of this paper is organized as follows. We will discuss related literature at the end 
of this section. Section 2 lays out the basic model. Section 3 analyzes objective IPE contracts and evaluates their value; an objective IPE is an IPE that is verifiable and this comes naturally as the first step of our analysis. Sections 4 analyzes the same for subjective IPE contracts and evaluates their value. Section 5 discusses and deals with some extensions. Section 6 concludes.

Related Literature This paper is related to a nascent and growing literature on IPE. Lizzeri, Meyer and Persico (2002) pioneer this research by studying how the (objective) IPE impacts the incentive of the agent. In a model where the outputs are independently generated at each stage, they show that the principal is hurt by conducting an IPE if she plans to induce any given level of efforts. By presenting another model, Ray (2007a) finds that IPE enhances efficiencies by providing the choice of ending the projects with low early returns. His key assumptions were that the production is indivisible and efforts across stages are perfect substitutes. In contrast with these two papers, our model assumes complementarity between the efforts across stages, and identifies a different trade-off of conducting IPE: the interim feedback, once the principal makes use of it in planning or control, would affect the agent's morale. Furthermore, by stressing the subjectivity of evaluation, we characterize the conditions under which contracting with or without IPE might be the optimal strategy.

In another related paper, Manso (2010) shows that when implementing a task with inter-period complementarity (such as "exploration"), the principal should provide (objective) feedback to the agent; but when implementing a task without such interdependence (such as "exploitation"), not providing the feedback is the optimal strategy. ${ }^{1}$ Since our main concern is the desirability of providing subjective feedback, which is less beneficial than the objective feedback due to the extra difficulty of information disclosure, Manso's analysis justifies our focus on a model with complementary tasks. In other words, Manso's finding obviates the need to study the subjective IPE in a model with independent tasks, because such evaluation is always undesirable.

Midterm reviews have also received significant notice in the recent literature on dynamic tournament. Some of these papers, including Yildirim (2005), Aoyagi (2008), Goltsman and Mukherjee (2009), Ederer (forthcoming), try to characterize the optimal strategy of interim information disclosure in the context of a two-stage tournament. Moreover, Gershkov and Perry (2009), while keeping

\footnotetext{
${ }^{1}$ According to Manso (2010), under "exploration", the probability of success in the second period is higher if the experimentation in the first period is successful; however, under "exploitation", the probability of success in each period is independent.
} 
a fixed disclosure policy, investigate the optimal aggregation and compensation rules in a tournament with or without midterm reviews. However, our model is built upon the standard principal-agent setting, instead of with multiple-agent competition. Another difference is that the contracting problem in our model goes hand in hand with information disclosure and morale formation.

Another relevant strand of literature is on subjective evaluation. By assuming the exogenous correlation between the principal's and the agent's beliefs, MacLeod (2003) finds that subjective evaluation results in the compression of performance levels. Motivated by relational contracts, Levin (2003) argues that if a subjective evaluation reveals low performance, the long-run relationship should be terminated in order to alleviate the moral hazard problem on the part of the agent and simultaneously prevent the adverse selection problem on the part of the principal. ${ }^{2}$ In contrast, we take a closer look at the subjective evaluation on the interim product, instead of the final product. The most distinctive feature is that both the principal's and the agent's beliefs of the project potential are reshaped by such an appraisal. For example, a negative feedback not only causes the agent's morale to drop, but also hinders the principal from further inducing efforts. In this sense, surplus burning is a natural consequence of the midterm review rather than something by construction.

\section{The model}

An agent is hired by a principal to complete a two-stage project. In Stage 1, the agent exerts an unobservable effort $e_{1} \in\{0,1\}$ with a cost $c_{1} e_{1}$, where $c_{1}>0$; at the end of the stage, an interim product of quality $x_{1} \in\{0,1\}$ is generated and the quality is high (i.e., $x_{1}=1$ ) with probability $r_{0}+r_{1} e_{1}$ or low (i.e., $\left.x_{1}=0\right)$ with the remaining probability, where $r_{0} \in(0,1)$ and $r_{1} \in\left(0,1-r_{0}\right)$. In Stage 2, the agent can make another unobservable effort $e_{2} \in\{0,1\}$ with a cost $c_{2} e_{2}$, where $c_{2}>0$; at the end of the stage, a final product is generated with quality $x_{2} \in\{0,1\}$.

If the interim product is of high quality, the final product is a "good" (i.e., $x_{2}=1$ ) with probability $t_{0}+t_{1} e_{2}$ or a "bad" (i.e., $x_{2}=0$ ) with the remaining probability, where $t_{0} \in(0,1)$ and $t_{1} \in\left(0,1-t_{0}\right)$; if the interim product is of low quality, however, the final product is a "good" with probability $t_{0}^{\prime}+t_{1}^{\prime} e_{2}$ or a "bad" with the remaining probability, where $t_{0}^{\prime} \in(0,1)$ and $t_{1}^{\prime} \in\left(0,1-t_{0}^{\prime}\right)$. The definition a high-quality interim product We assume that $t_{0} \geq t_{0}^{\prime}$ to accord with the definition of a high-quality interim product and that $t_{1}>t_{1}^{\prime}$ so that there is complementarity between efforts

\footnotetext{
${ }^{2}$ Fuchs (2007) extends the analyses of MacLeod (2003) and Levin (2003) to a dynamic environment, and characterizes the optimal timing of evaluation and the optimal rule of termination.
} 
across stages. ${ }^{3}$

The nature of the final product is observable and verifiable, and the principal gains from the project a value of $B>0$ in the case of a final "good" and a value of zero in the case of a final "bad." We assume that both the principal and the agent are risk-neutral, and that there is no discounting across stages. However, the agent has no wealth and is protected by limited liability; his reservation utility is assumed to be zero.

Although not immediately observable, $x_{1}$ can be learned through an IPE. Specifically, subsequent to its realization but prior to Stage 2, the principal has an option of arranging an evaluation through which she obtains a signal $\sigma \in\{H, L\}$ that perfectly reveals $x_{1}$. Projects fitting our model can be found in many industries such as construction and software development. The potential safety problems of bridges under construction or possible bugs in an unfinished software are not easily known unless the project manager undertakes interim checkups. On the properties of evaluation, we make two assumptions for simplicity. First, we assume that the cost of the evaluation is zero. Obviously, any positive cost will simply reduce the net gains of doing the IPE by the same amount. Second, we assume the evaluation contains no noise; at the end of this paper, we will discuss the impacts of a noisy IPE on the optimal contracting.

The timing of this game is summarized in Figure 1. At the outset, the principal determines whether to conduct an IPE, and proposes and signs a take-it-or-leave-it contract with the agent. The production process goes through Stage 1 and Stage 2 sequentially. In case an IPE is scheduled, it will take place at the end of Stage 1 , followed by a feedback $m$ provided by the principal to the agent.

In case an IPE is not scheduled, the contract will only be contingent on the verifiable nature of the final product, $x_{2}$. So it is represented by a duple $(w, b)$, where $w$ is a wage rate that must be

\footnotetext{
${ }^{3}$ We could show that, in this model, if $t_{1}<t_{1}^{\prime}$, the efforts between the two stages are substitutes; if $t_{1}=t_{1}^{\prime}$, the two-stage efforts are independent; if $t_{1}>t_{1}^{\prime}$, the efforts between the two stages are complements. In our model, the complementarity may result from the fact that satisfactory completion of previous phases lays the foundation for proceeding to the next phase; Manso (2010) also argues that complementarity naturally arises when the principal tries to design a contract for "exploration."
} 


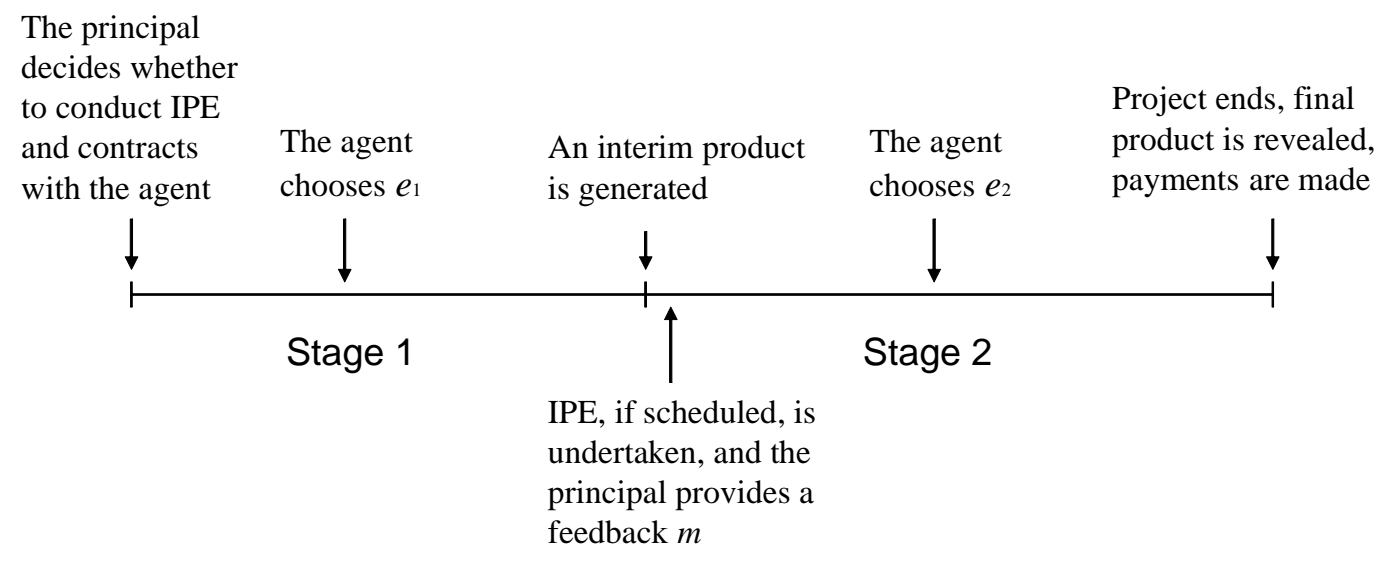

Figure 1: The timeline of the game with the option of an IPE. 
paid out and $b$ is a bonus paid out if and only if $x_{2}=1$. The agent's limited liability dictates that ${ }^{4}$

$$
w \geq 0 ; b+w \geq 0
$$

We call such a contract a "traditional contract." We use $\left\langle e_{1} ; e_{2}\right\rangle$ to denote the corresponding action plan, where $e_{1}$ and $e_{2}$ are the agent's efforts in Stage 1 and Stage 2.

In case an IPE is scheduled, the choice of contract is enriched because it could be written contingent not only on $x_{2}$ but also on the interim feedback $m$ (disclosed by the principal). There are two scenarios: the IPE may be objective so that the IPE outcome, denoted by $\sigma$, is publicly observable and verifiable, or it may be subjective so that $\sigma$ is the principal's private information. Without loss of generality, for both types of IPEs, we assume that the set of all possible messages that the principal may utter equals $\{H, L\}$. Thus, the contract is represented by a quadruple $\left(w_{H}, w_{L}, b_{H}, b_{L}\right)$, where, given feedback $m, w_{m}$ is the wage rate paid out to the agent regardless of $x_{2}$ and $b_{m}$ is the additional bonus paid out if $x_{2}=1$. The limited liability constraints dictate that

$$
w_{H}, w_{L} \geq 0 ; \quad b_{H}+w_{H} \geq 0 ; \quad b_{L}+w_{L} \geq 0 .
$$

We call such a contract a "milestone contract." Note that the Stage-2 effort would be conditional on the feedback; we denote the corresponding implemented action plan as $\left\langle e_{1} ; e_{2}(H), e_{2}(L)\right\rangle$. To facilitate our exposition, we make the following definition.

Definition 1 A milestone contract is called an effort-sorting scheme if it implements the action plan $\langle 1 ; 1,0\rangle$.

Given contract $\phi$, we use $V^{\phi}$ to denote the expected revenue accruing to the principal, $C^{\phi}$ to denote the expected cost she incurs, and $\pi^{\phi}$ to denote her expected payoff; so we have $\pi^{\phi} \equiv V^{\phi}-C^{\phi}$.

Without the IPE, our model is just a variant of the hidden-action model with limited liability, which is a building block of many recent papers on the agency problem (e.g., Crémer 1995, Che and Yoo 2001, Schmitz 2005, etc.). In the context of procurement and project management, the assumption of limited liability is quite reasonable because the agents are usually protected by bankruptcy

\footnotetext{
${ }^{4}$ We implicitly assume that $w$ is paid out along with $b$ (if rewarded) at the very end. It is possible that $w$ is paid out at the interim, whereas $b$ is paid out at the end of the project. In this case, one may consider a more restrictive limitedliability constraint, which requires that $b$ be non-negative. As later analysis will show, this alternative treatment of limited liability will not change any results, because the optimal choice of $b$ in any contract constrained by the less restrictive limited-liability constraints (e.g., (1) or (2)) is not lower than 0.
} 
laws (see, e.g., Calveras, Ganuza and Hauk 2004). Like Schmitz (2005), we make the following assumption, in order to simplify the exposition and avoid tedious case distinctions.

Assumption 1.

$$
\frac{c_{1}}{c_{2}} \leq \min \left\{\frac{r_{1} t_{0}}{t_{1}}, \frac{r_{1}\left(t_{0}-t_{0}^{\prime}\right)}{\left(r_{0}+r_{1}\right) t_{1}+\left(1-r_{0}-r_{1}\right) t_{1}^{\prime}}\right\}
$$

and

$$
B \geq \max \left\{\underline{B}^{\prime}, \underline{B}^{\prime \prime}\right\}
$$

where

$$
\begin{aligned}
\underline{B}^{\prime} \equiv & \frac{\left[\left(r_{0}+r_{1}\right)\left(t_{0}+t_{1}\right)+\left(1-r_{0}-r_{1}\right)\left(t_{0}^{\prime}+t_{1}^{\prime}\right)\right] c_{2}}{\left[\left(r_{0}+r_{1}\right) t_{1}+\left(1-r_{0}-r_{1}\right) t_{1}^{\prime}\right]^{2}} \\
& -\frac{\left[\left(r_{0}+r_{1}\right) t_{0}+\left(1-r_{0}-r_{1}\right) t_{0}^{\prime}\right] c_{1}}{r_{1}\left(t_{0}-t_{0}^{\prime}\right)\left[\left(r_{0}+r_{1}\right) t_{1}+\left(1-r_{0}-r_{1}\right) t_{1}^{\prime}\right]}, \text { and } \\
\underline{B}^{\prime \prime} \equiv & \frac{\left[\left(r_{0}+r_{1}\right)\left(t_{0}+t_{1}\right)+\left(1-r_{0}-r_{1}\right)\left(t_{0}^{\prime}+t_{1}^{\prime}\right)\right] c_{2}}{\left[\left(r_{0}+r_{1}\right) t_{1}+\left(1-r_{0}-r_{1}\right) t_{1}^{\prime}\right]} \\
& \times \frac{1}{\left\{\left[\left(r_{0}+r_{1}\right)\left(t_{0}+t_{1}\right)+\left(1-r_{0}-r_{1}\right)\left(t_{0}^{\prime}+t_{1}^{\prime}\right)\right]-\left[r_{0} t_{0}+\left(1-r_{0}\right) t_{0}^{\prime}\right]\right\}} .
\end{aligned}
$$

In particular, the assumption guarantees that the principal will implement $\langle 1 ; 1\rangle$ in absence of any IPE, and that the agent, potentially shirking, is more likely to shade his effort in Stage 2 than in Stage 1. If Assumption 1 is not satisfied, the analysis is analogical to what follows. However, no additional insights are gained. (Note that the IPE is more likely to influence the belief and incentive of the agent in the post-evaluation stage. So the assumption restricts attention to the most interesting case, in which the Stage-2 Incentive Compatible constraint is more likely to be binding. We will come back to discuss what happens if $c_{1} / c_{2}$ becomes large.)

Under Assumption 1, the optimal traditional contract should implement $\langle 1 ; 1\rangle$. Denote the contract by $T$. One can easily verify that $w^{T}=0$, and

$$
b^{T}=\frac{c_{2}}{\left(r_{0}+r_{1}\right) t_{1}+\left(1-r_{0}-r_{1}\right) t_{1}^{\prime}}
$$

with corresponding implementation cost

$$
C^{T}=\frac{\left[\left(r_{0}+r_{1}\right)\left(t_{0}+t_{1}\right)+\left(1-r_{0}-r_{1}\right)\left(t_{0}^{\prime}+t_{1}^{\prime}\right)\right] c_{2}}{\left(r_{0}+r_{1}\right) t_{1}+\left(1-r_{0}-r_{1}\right) t_{1}^{\prime}}
$$




\section{Objective milestone contract}

In this section we study the scenario in which the IPE is objective. In this case, the message uttered by the principal will be the same as the IPE outcome, and there is no incentive problem whether the principal will lie or not. Before discussing any specific contract, we show the following property.

Lemma 1 Suppose that the IPE is objective. For any action plan, to characterize the optimal contract, it is without loss of generality focusing on the case where $w_{L}=w_{H}=0$.

Holding $w_{H}, b_{L}$, and $b_{H}$ constant, an increase in $w_{L}$ leads to a higher expected cost to be paid out, while also weakening the agent's incentive to exert effort in the first stage. It is thus undesirable. In our setting, $w_{H}$ and $b_{H}$ are equally effective in making the first stage IC constraint bind. However, it can be shown that, however, when the IPE outcome contains noisy signal about the quality of the intermediate good, than using $b_{H}$ is more cost effective than using $w_{H}$. The reason is that the signal generated by the IPE cannot be a more precise indicator of the final product than the final production itself, and that setting a reward contingent on $\sigma=H$ alone (e.g., using wages) is less desirable than deferring such a reward until the whole project is successfully completed (e.g., using bonus). ${ }^{5}$ Therefore, focusing on the case where $w_{H}=0$ is not only without loss of generality, but also is also robust to the introduction of some small noise to the IPE outcome.

In what follows, we turn to the optimal milestone contracts that implement $\langle 1 ; 1,1\rangle$ and $\langle 1 ; 1,0\rangle$, denoted by $O 1$ and $O 0$, respectively.

\subsection{Implementing $\langle 1 ; 1,1\rangle$}

We now characterize $O 1$. Consider the agent's second stage decision. Given a $H$ signal (hence the same message uttered by the principal), the agent prefers choosing $e_{2}=1$ to choosing $e_{2}=0$, if and only if

$$
b_{H} \geq \frac{c_{2}}{t_{1}},
$$

where $t_{1} b_{H}$ is the expected benefit of exerting stage- 2 effort and $c_{2}$ is the cost of the effort. This condition is in fact a very familiar equation in moral hazard problem with limited liability constraint.

\footnotetext{
${ }^{5}$ Consider the extreme case in which the IPE signal is $H$ with probability $1 / 2$ regardless of the quality of the interim product. Then while increasing the cost to the principal, any increase in $w_{H}$ will have no effect in motivating the agent to exert $e_{1}=1$. On the other hand, an increase in $b_{H}$ will certainly make choosing $e 1=1$ more attractive for the agent.
} 
In a similar token, given a $L$ signal, the agent prefers choosing $e_{2}=1$ to choosing $e_{2}=0$, if and only if

$$
b_{L} \geq \frac{c_{2}}{t_{1}^{\prime}}
$$

Notice that, because $t_{1}>t_{1}^{\prime}$, the minimum bonus that motivates the agent to work hard in stage 2 is greater when $L$ signal is received. The intuition is that now that the agent has low morale, or is less confident about the project, he has to be given greater incentive.

The last IC constraint to check is that the agent has no deviation motive in stage 1 . At the outset, foreseeing that he will choose $e_{2}=1$ for certainty, the agent prefers choosing $e_{1}=1$ to choosing $e_{1}=0$ if and only if

$$
r_{1}\left(t_{0}+t_{1}\right) b_{H}-r_{1}\left(t_{0}^{\prime}+t_{1}^{\prime}\right) b_{L}+r_{1}\left(w_{H}-w_{L}\right) \geq c_{1} .
$$

Notice that an increase in $b_{L}$ makes this condition more difficult to hold. Taken into account $w_{H}=w_{L}=0$, the condition is found to be equivalent to

$$
b_{H} \geq \frac{c_{1}}{r_{1}\left(t_{0}+t_{1}\right)}+\frac{\left(t_{0}^{\prime}+t_{1}^{\prime}\right) c_{2}}{\left(t_{0}+t_{1}\right) t_{1}^{\prime}}
$$

In case this minimum $b_{H}$ exceeds that one calculated in (5), then the purpose of $b_{H}$ is to motivate stage 1 effort, rather than to motivate stage 2 effort under $H$ signal. ${ }^{6}$ It should be noticed that Assumption 1 itself does not preclude the possibility of the RHS of (8) exceeding the RHS of (5). It suggests a potential cost of using IPE because now additional constraints need to be satisfied. The following proposition summarizes the characterization of this IPE contract.

Proposition 1 Suppose that the IPE is objective. O1 satisfies $w_{H}=w_{L}=0, b_{L}=c_{2} / t_{1}^{\prime}$ and

$$
b_{H}=\max \left\{\frac{c_{2}}{t_{1}}, \frac{c_{1}}{r_{1}\left(t_{0}+t_{1}\right)}+\frac{\left(t_{0}^{\prime}+t_{1}^{\prime}\right) c_{2}}{\left(t_{0}+t_{1}\right) t_{1}^{\prime}}\right\} \cdot 7
$$

We now examine if $O 1$ is more profitable than $T$ is. Because both $O 1$ and $T$ correspond to the same action plan (hence the same expected revenue), the question becomes whether $O 1$ leads

\footnotetext{
${ }^{6}$ It can be shown that any other IC constraints are satisfied so long as the three aforementioned three IC constraints are satisfied.

${ }^{7}$ In case the second term in the RHS of (9) is greater, any contract that satisfies $w_{L}=0, b_{L}=c_{2} / t_{1}^{\prime}, b_{H} \in$ $\left[\frac{c_{2}}{t_{1}}, \frac{c_{1}}{r_{1}\left(t_{0}+t_{1}\right)}+\frac{\left(t_{0}^{\prime}+t_{1}^{\prime}\right) c_{2}}{\left(t_{0}+t_{1}\right) t_{1}^{\prime}}\right]$ and $w_{H}=\frac{c_{1}}{r_{1}}+\frac{\left(t_{0}^{\prime}+t_{1}^{\prime}\right) c_{2}}{t_{1}^{\prime}}-\left(t_{0}+t_{1}\right) b_{H}$ is outcome equivalent and is also optimal.
} 
to lowering of the expected cost. Denote the cost under $O 1$ and $T$ by $C^{O 1}$ and $C^{T}$, respectively. Defining $\pi_{H}=\left(r_{0}+r_{1}\right)\left(t_{0}+t_{1}\right)$ and $\pi_{L}=\left(1-r_{0}-r_{1}\right)\left(t_{0}^{\prime}+t_{1}^{\prime}\right)$, then we have

$$
C^{O 1}=\pi_{H} b_{H}+\pi_{L} b_{L} \text { and } C^{T}=\pi_{H} b^{T}+\pi_{L} b^{T} .
$$

To fix idea, suppose $b_{H}$ indeed equals $c_{2} / t_{1}$ (so that the IC constraint that prevents deviation to $\langle 0 ; 1,1\rangle$ is nonbinding, or (8) can be ignored). Then we have $b_{H}<b^{T}<b_{L}$. Hence, a switching from $T$ to $O 1$ results in a reduction of cost when $H$ is observed (beneficial effect) but an increase in cost when $L$ is observed (harmful effect). $O 1$ leads to a greater agency cost if the latter, harmful effect is dominant. Notice that, as $t_{1}^{\prime}$ decreases, $b_{L}$ increases without a finite upper bound (while $b_{H}$ remains unchanged and $b^{T}$ increases with a finite upper bound). Hence, the harmful effect must be dominant for sufficiently small $t_{1}^{\prime}$, and one can easily find parameter values such that $O 1$ increases the agency cost. What is more interesting is we can also find parameter values such that the converse is true. The following proposition formally states the result.

Proposition 2 1. Suppose $\frac{t_{1}^{\prime}}{t_{0}^{\prime}}<\frac{t_{1}}{t_{0}}$. Then the implementation cost of $O 1$ is strictly greater than that of $T$.

2. Suppose $\frac{t_{1}^{\prime}}{t_{0}^{\prime}}>\frac{t_{1}}{t_{0}}$. Then there exists $R^{*}>0$ such that for all $c_{1}<R^{*} c_{2}$, the implementation cost of $O 1$ is strictly lower than that of $T$.

Notice that $t_{1}^{\prime} / t_{0}^{\prime}\left(t_{1} / t_{0}\right)$ is the ratio of additional success probability over the default success probability of stage 2's effort given signal $L$ (signal $H$ ). Result 1 states that if this ratio is lower under signal $L$ than under signal $H$, it is not worthwhile conducting IPE. The intuition is that it is too costly to motivate an agent under signal $L$ (or the limited liability rent under IPE is too large). Result 2 states that, if the ratio is greater under signal $L$ than under signal $H$, it may be worthwhile conducting IPE. ${ }^{8}$ The restriction to $c_{1}<R^{*} c_{2}$ is to make sure that $b_{H}$ indeed equals $c_{2} / t_{1}$ and the IC constraint that prevents deviation to $\langle 0 ; 1,1\rangle$ is nonbinding. In this case, doing IPE is beneficial. In case the IC constraint is binding, however, the $b_{H}$ exceeds $c_{2} / t_{1}$ and the cost $C^{O 1}$ calculated above assuming $b_{H}=c_{2} / t_{1}$ is an underestimation of the true cost. Beneficial IPE is not guaranteed.

To summarize, there are two reasons why $C^{O 1}$ may exceed $C^{T}$. First, the increase in bonus

\footnotetext{
${ }^{8}$ We also know from the literature on agency problem with limited liability (e.g., Schmitz 2005) that the rent is positively related to the default probability of success and negatively related to the success probability added by effort, so the ratios of $t_{0} / t_{1}$ and $t_{0}^{\prime} / t_{1}^{\prime}$ dictate the limited liability rent by revealing $H$ and $L$, respectively.
} 
under $L$ signal is too high for the reduction in bonus under $H$ signal to fully compensate. Second, the reduction of bonus under $H$ signal may be not as large as initially expected; the result is that, compared with traditional contracting, additional IC constraints are introduced in $C^{O 1}$ and some of them may require a $b^{H}$ greater than is required solely to motivate the stage 2 effort under $H$ signal.

\subsection{Implementing $\langle 1 ; 1,0\rangle$}

We now characterize $O 0$. Like $O 1, O 0$ should still prescribe that $w_{H}=w_{L}=0 . b_{L}$ should be made as low as zero because, given action plan $\langle 1 ; 1,0\rangle$, there is no need to motivate the agent to work hard when knowing $\sigma=L . b_{H}$ should still satisfy (5) so that the agent will not deviate to $e_{2}=0$ when knowing $\sigma=H$. The last thing to check is that at the outset the agent has no incentive to deviate to action plan $\langle 0 ; 1,0\rangle$. This condition, after plugging $w_{H}=w_{L}=0$ (from Lemma 1 ) and $b_{L}=0$ (obviously the case), is thus equivalent to

$$
b_{H} \geq \frac{c_{1}+r_{1} c_{2}}{r_{1}\left(t_{0}+t_{1}\right)}
$$

Thus $b_{H}$ should be the maximum of this term and $c_{2} / t_{1}$. But, interestingly, this term is always less than $c_{2} / t_{1}$ given Assumption $1 .^{9}$ As a result, $O 0$ is characterized as follows:

Proposition 3 Suppose that the IPE is objective. O0 is characterized by $w_{H}=w_{L}=b_{L}=0$ and $b_{H}=\frac{c_{2}}{t_{1}}$.

The comparison of agency cost between $O 0$ and $T$ is fairly straightforward.

Proposition 4 There exists a cutoff $B^{O}$ such that O0 yields a higher (lower) level of profit to the principal than $T$ if $B<B^{O}\left(B>B^{O}\right)$. Moreover, this cutoff $B^{O}$ is increasing in $c_{2}, t_{0}^{\prime}$, and $t_{0}$, and decreasing in $t_{1}^{\prime}$.

The intuition is straightforward. Compared with $T$, the effort sorting scheme $O 0$ brings in a smaller revenue but also incurs lower costs. While the reduction in revenue is propositional to $B$, the reduction in costs is independent of $B$. Thus, $O 0$ is attractive only when the first (detrimental) effect is low enough, i.e., when the project value $B$ is low enough.

\footnotetext{
${ }^{9}$ The RHS of (10) is smaller than the RHS of (8). In other words, the incentive to shirk in the first stage is lower under $O 0$ than under $O 1$. The reason is as follows. Under 01 , the agent, even after shirking in stage 1 , will still be induced to work hard under L-signal, via an attractive bonus $b_{L}=c_{2} / t_{1}^{\prime}>c_{2} / t_{1}$. Under O0, no such incentive is available under $L$-signal. As a result, it becomes more attractive for the agent to shirk under $O 1$ than under $O 0$.
} 
The comparative statics state that $O 0$ is more desirable, if the ratio of $t_{0}^{\prime} / t_{1}^{\prime}$ is higher. A larger ratio of $t_{0}^{\prime} / t_{1}^{\prime}$ is associated with greater limited liability rents, when inducing continuation effort upon an interim product of low quality. The traditional contract $T$ does not rely on an IPE, but indeed induces $e_{2}=1$ upon an interim product of low quality. Hence, a rise in the ratio of $t_{0}^{\prime} / t_{1}^{\prime}$ increases the average rent paid to the agent. However, the effort-sorting scheme, by ceasing to inducing any effort after revealing $L$, does not suffers from this problem. Thus a rise in the ratio of $t_{0}^{\prime} / t_{1}^{\prime}$ favors the use of effort sorting. ${ }^{10}$ The comparative statics with respect to $t_{0}$ is due to fact that a greater $t_{0}$ leads to greater rents when $H$ signal is observed. Although $C^{O 0}$ and $C^{T}$ increase in $t_{0}$, the latter increases more rapidly in $t_{0}$; the reason is that when working upon an interim product of high quality, the bonus, as well as limited liability rent, is reduced if a positive feedback is provided. ${ }^{11}$

\subsection{The value of IPE contracts}

Thus far, we have characterized $O 1$ and $O 0$ and compared each with respect to $T$. What remains to be done is the find out the optimal contract when IPE option is available but need not be adopted in the contract. A second question is how this optimal contract performs with respect to the first best.

For both questions, we can put down our analysis using a graphical method (refer to figure 2). The horizontal axis ist $t_{1}^{\prime}$, over which our attention is that $t_{1}^{\prime}<t_{1}$ so that efforts are complementarity. The vertical axis is $B$ and we focus on the range that assumption 1 is satisfied, and we assume other parameters $\left(c_{1}\right.$, and $\left.c_{2}\right)$ are unchanged in the exercise. The whole space in the figure thus represents the parameter range over which $T$ is the optimal traditional contract.

Optimal contract Given Assumption 1, we can verify that the optimal IPE contract would be

either $O 1$ or $O 0 .{ }^{12}$ Notice that between these two contracts, the principal will choose the former

\footnotetext{
${ }^{10} \mathrm{~A}$ smaller $t_{1}^{\prime}$ not only enlarges the saving on the limited liablity rent but also reduces the expected revenue loss of effort sorting.

${ }^{11}$ The comparative static with respect to to $t_{1}$ is more complicated, because it also determines the degree of complementarity between efforts across stages.

${ }^{12}$ Other action plans are not optimal. The reasons are as follows. First, inducing $\langle 1 ; 0,1\rangle$ is less desirable than inducing $\langle 1 ; 1,0\rangle$. The latter allows the principal to not only reap a greater expected revenue but also curtail a greater expected cost, because the agent is now motivated by an optimistic belief to choose a high Stage-2 effort. Second, inducing $\langle 1 ; 0,0\rangle$ is less desirable than inducing $\langle 1 ; 1,1\rangle$, simply because traditional contract implementing $\langle 1 ; 0\rangle$ is dominated by that implementing $\langle 1 ; 1\rangle$ given Assumption 1. Third, inducing a low effort in Stage 1, e.g., the action plan $\langle 0 ; y, z\rangle$, is never optimal; this is further guaranteed by Assumption 1: when $c_{1} / c_{2}$ is sufficiently low and the project is sufficiently valuable, the principal will not benefit from a low Stage-1 effort.
} 


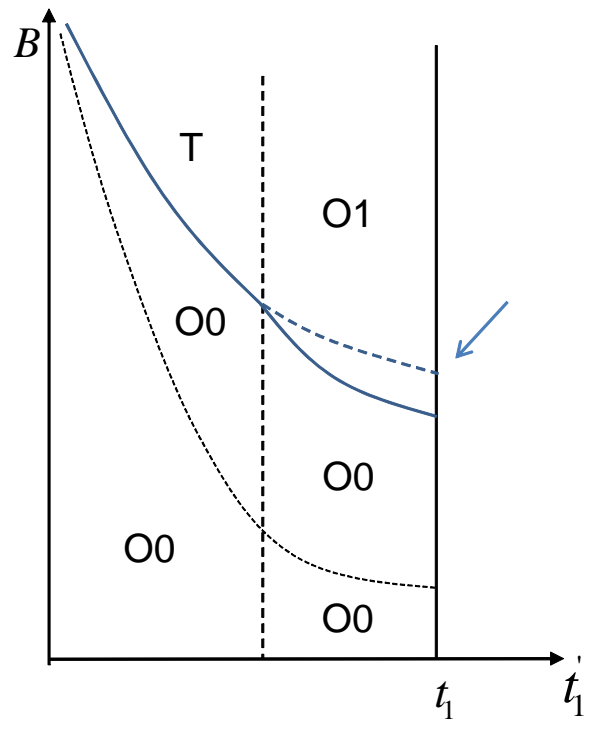

Panel a: principal's optimal contract

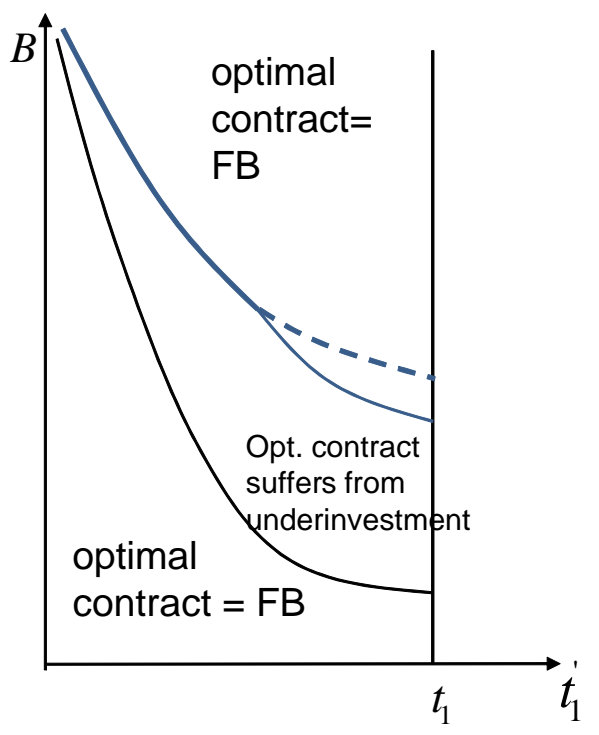

Pane b: comparison between optimal contract and first best

Figure 2: optimal contract and its comparison with the first best 
over the latter if its extra benefit more than offsets its extra cost, which is equivalent to

$$
B>\widetilde{B} \equiv \frac{t_{0}^{\prime}+t_{1}^{\prime}}{\left(t_{1}^{\prime}\right)^{2}} c_{2}
$$

This $\widetilde{B}$ is decreasing in $t_{1}^{\prime}$, intersecting $B^{O}$ from above when $t_{1}^{\prime}=\left(t_{0}^{\prime} / t_{1}^{\prime}\right) t_{1}$. Thus with the help of our earlier propositions, the space can be partitioned into four regions, using the vertical line $t_{1}^{\prime}=\left(t_{1}^{\prime} / t_{0}\right) t_{1}$ and the downward slopping line $B^{O}$. In the bottom left region, the optimal contract is $O 0$; in the top left region, the optimal contract is $T$; in the top right region, the optimal contract is $O 1$; finally, in the bottom right region, the optimal contract is $O 0$. This thus completely characterizes the optimal contract when IPE is available and need not be chosen.

comparison with first best Now we turn to the comparison with the first best. Note that given assumption 1 , the first best dictates that either $\langle 1 ; 1,1\rangle$ or $\langle 1 ; 1,0\rangle$ will be implemented. The former is chosen over the latter iff its extra benefit more than offsets its extra cost. The condition is equivalent to

$$
B>B^{*} \equiv \frac{c_{2}}{t_{1}^{\prime}},
$$

which is a downward slopping line in the Figure. One can easily show that $B^{*}<\widetilde{B}$ and $B^{*}<B^{O}$. As a result, the space can be divided into three bands. In the top and bottom bands, the principal's choice is as efficient as the first best. In the middle band, the principal implements $\langle 1 ; 1,0\rangle$ while the first best is to implement $\langle 1 ; 1,1\rangle$ so there is an underinvestment problem and the availability of IPE reduces efficiency compared with traditional contracting. In the bottom band, the principal's choice accords with the first best so the availability of IPE improves efficiency compared with traditional contracting.

\subsection{Relationship with the literature}

We end with some discussion with the literature. According to Ray (2007a) and Manso (2010), providing the (objective) feedback is beneficial for the principal, if it helps her screen out bad projects or provide proper incentives for "exploration." Such advantage of IPE can be translated into the implementation of "effort-sorting" scheme in our setting: the conduct of IPE can let the Stage-2 effort contingent on the Stage-1 outcome. Moreover, we find that the effort-sorting scheme 
payoff dominates the traditional contract only under certain condition, which is favored by a smaller $B$ or a larger ratio of $t_{1}^{\prime} / t_{0}^{\prime}$.

However, our finding contrasts with Lizzeri, Meyer and Persico (2002), in the sense that for inducing any given action plan, the revelation of feedback can reduce the implementation cost under some condition, instead of enhancing the cost always. The difference is caused by the fact that we assume the complementarity between efforts across stages. We indeed consider a more general framework, by emphasizing the ratio of success probability that is key to the agency problem with limited liability. The linkage between our model and Lizzeri, Meyer and Persico (2002) can also be

established: if $t_{1}^{\prime}$ is larger than $\frac{t_{0}^{\prime}}{t_{0}} t_{1}$, we find that $O 1$ payoff dominates $T$. However, if $t_{1}^{\prime}$ increases close to $t_{1}$, the Stage- 1 and Stage- 2 efforts tend to be independent. In this case, the cost advantage of $O 1$, relative to $T$, vanishes.

\section{Subjective milestone contract}

In this section we study the scenario in which the IPE is subjective. The principal is now tempted to lie or to hide the signal from the agent and hence the contracting problem is complicated by this adverse selection problem. We focus on milestone contracts with the following full-revealing property.

Definition 2 A milestone contract is said to satisfy the full-revealing property, if the message $m$ announced by the principal is consistent with the true signal $\sigma$ learnt in the subjective IPE.

In other words, the new contracting problem is now constrained by additional "truth-telling" conditions. We focus on the optimal milestone contracts that implement $\langle 1 ; 1,1\rangle$ and $\langle 1 ; 1,0\rangle$; we denote the two contracts by S1 and S0, respectively.

\subsection{Implementing $\langle 1 ; 1,1\rangle$}

To implement $\langle 1 ; 1,1\rangle$, the cost-minimization problem confronting the principal is the same as the case of objective IPE, except that the following two truth-telling constraints are added.

$$
\begin{aligned}
& \left(t_{0}+t_{1}\right)\left(b_{L}-b_{H}\right) \geq w_{H}-w_{L} ; \\
& \left(t_{0}^{\prime}+t_{1}^{\prime}\right)\left(b_{L}-b_{H}\right) \leq w_{H}-w_{L} .
\end{aligned}
$$


(11) states that, even if the agent was to believe in the principal, the principal would not benefit from lying to have received signal $L$ when she had actually received $H ;(12)$ is the corresponding condition so that the principal would not benefit from lying to have received signal $H$ when she had actually received $L$. We can verify that, given contract $O 1,(12)$ is violated and (11) is not ; i.e., the principal will have an incentive to lie when receiving $L$ signal but no incentive to lie when receiving $H$ signal.

As it will be shown, the optimal contract will still entail $w_{L}=0$ and $b_{L}=\frac{c_{2}}{t_{1}^{\prime}}$; an increase in $w_{L}$ or $b_{L}$ will only increase the expected cost and worsen the IC constraint (12). What may be different are the choices of $w_{H}$ and $b_{H}$. For that purpose, we can represent their choices in a $\left(b_{H}, w_{H}\right)$ diagram (see Figure 3$)$. (11) is satisfied for all $\left(b_{H}, w_{H}\right)$ below the $H$-line; (12) is satisfied for all $\left(b_{H}, w_{H}\right)$ above the $L$-line. Notice that both lines have a horizontal intercept of $c_{2} / t_{1}^{\prime}$. An additional constraint, $b_{H} \geq c_{2} / t_{1}$, is depicted which is to ensure $e_{2}=1$ upon the agent receiving a high message. Given that IC is always on the left hand side of the common horizontal intercept of the $H$-line and $L$-line, there always exists a shaded region over which all three constraints are satisfied. it is straightforward to verify that the optimal $\left(b_{H}, w_{H}\right)$ is the dotted point within the shaded region.

Proposition 5 Suppose $c_{1} / c_{2}$ is sufficiently small. Then $\langle 1 ; 1,1\rangle$ is always implementable and $S 1$ satisfies $w_{L}=0, b_{L}=\frac{c_{2}}{t_{1}^{\prime}}, b_{H}=c_{2} / t_{1} ;$ and $w_{H}=\left(t_{0}^{\prime}+t_{1}^{\prime}\right)\left(\frac{c_{2}}{t_{1}^{\prime}}-\frac{c_{2}}{t_{1}}\right)$.

The condition $c_{1} / c_{2}$ is to ensure that we can ignore the IC constraint that prevents deviation to action plan $\langle 0 ; 1,1\rangle$ so that the only relevant constraints are (5), (6), (11), and (12). Not only simplifying the characterization of $S 1$, this assumption also ensures that $\langle 1 ; 1,1\rangle$ is indeed implementable. When $c_{1} / c_{2}$ is not sufficiently small, $b_{H}$ may be made larger to ensure no deviation to action plan $\langle 0 ; 1,1\rangle$. That means the IC line will move rightward and hence the existence of the shaded region in Figure 3 is no longer guaranteed.

The characterization of $S 1$ has interesting properties. The contract is the same as $O 1$, except that $w_{H}$ is made large enough to ensure that the principal has no lying incentive when receiving a $L$ signal. In principle, her honesty can also be ensured with an increase in $b_{H}$ (or a simultaneous increase of both $b_{H}$ and $w_{H}$ ) but that is not most cost effective from the principal's point of view. The intuition is as follows. The $L$ line in the diagram has a slope of $t_{0}^{\prime}+t_{1}^{\prime}$ in absolute term. That is, the principal will remain to have no incentive to lie under signal- $L$ when $w_{H}$ is increased by one 


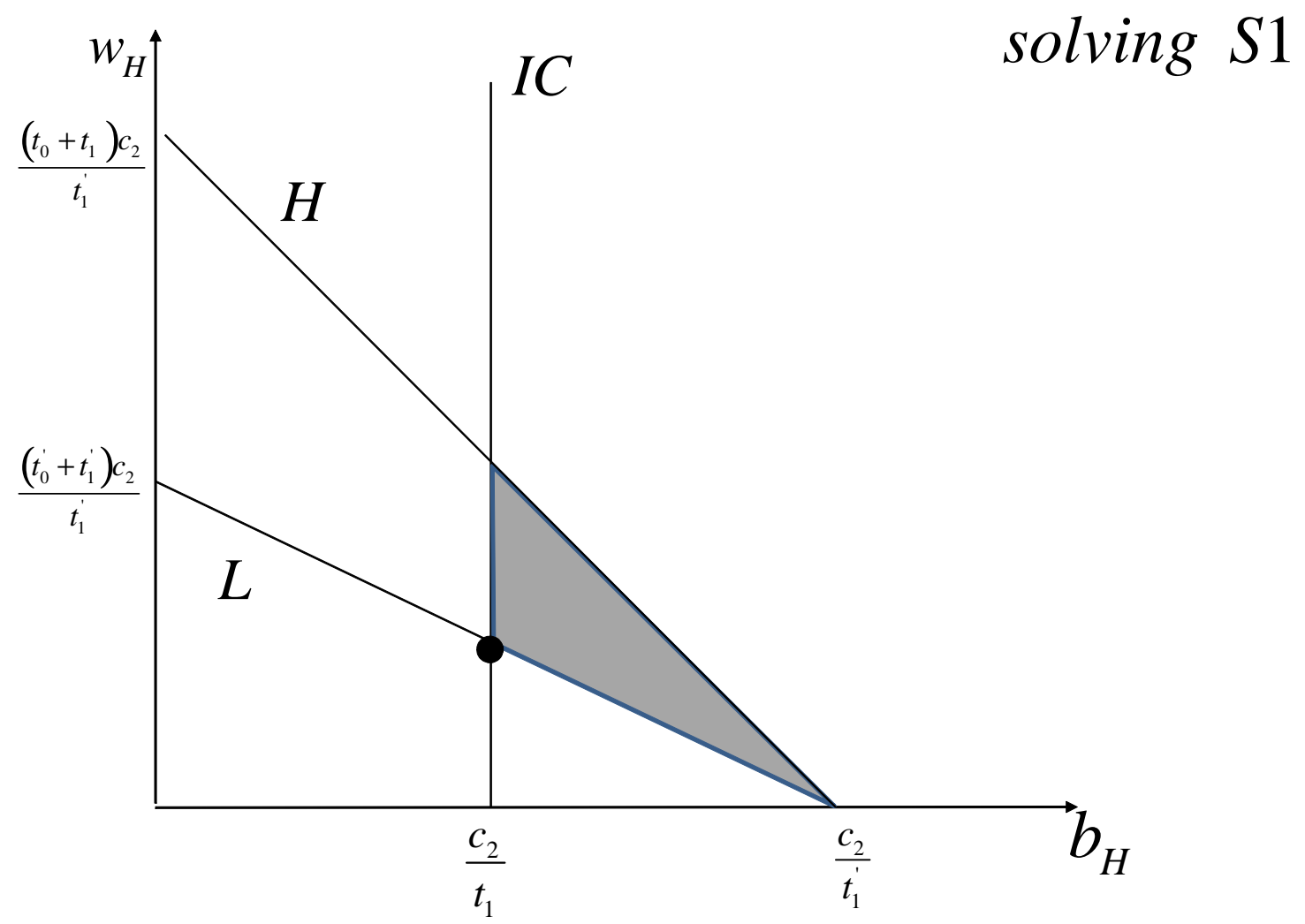

Figure 3: The value of subjective milestone contract 
and $b_{H}$ is reduced by $1 /\left(t_{0}^{\prime}+t_{1}^{\prime}\right)$. On the other hand, the principal's expected cost remains same when $w_{H}$ is increased by one unit and $b_{H}$ is decreased by $1 /\left(t_{0}+t_{1}\right)<1 /\left(t_{0}^{\prime}+t_{1}^{\prime}\right)$. This explains why using $w_{H}$ is more cost effective than using $w_{H}$.

It is less likely that $\langle 1 ; 1,1\rangle$ is implemented at a lower cost under $T$ when IPE is subjective. Despite its restrictiveness, beneficial $S 1$ is not impossible. The following proposition summaries this result.

Proposition 6 There exists parameters under which $S 1$ exists and the expected cost is lower than that under $T$.

\subsection{Implementing $\langle 1 ; 1,0\rangle$}

To implement $\langle 1 ; 1,0\rangle$, the cost-minimization problem confronting the principal is the same as the case of objective IPE, except that the following two truth-telling constraints are added.

$$
\begin{aligned}
& \left(t_{0}+t_{1}\right)\left(B-b_{H}\right)-w_{H} \geq t_{0}\left(B-b_{L}\right)-w_{L} \\
& t_{0}^{\prime}\left(B-b_{L}\right)-w_{L} \geq\left(t_{0}^{\prime}+t_{1}^{\prime}\right)\left(B-b_{H}\right)-w_{H} .
\end{aligned}
$$

(13) states that, even if the agent was to believe the principal, the principal would not benefit from lying to have received signal $L$ when she has actually received $H ;(14)$ is the corresponding condition so that the principal would not benefit from lying to have received signal $H$ when she has actually received $L$.

The analysis of the optimal IPE contract is similar is the case of $S 1$, using a graphical method. It is conceivable that $w_{L}=0$ and $b_{L}=0$ as in $O 0$; an increase in either of them would only increase the expected cost as well as making (14) more difficult to hold. Then the question boils down to finding a pair of $b_{H}$ and $w_{H}$. Using a graphical method as we did for $S 1$, we depict three constraints (5), (13), and (14). ${ }^{13}$ Notice that now in general $H$-line and $L$-line do not have a common horizontal intercept. When $\frac{t_{1}^{\prime}}{t_{0}^{\prime}}>\frac{t_{1}}{t_{0}}$, the $H$-line's horizontal intercept is smaller. A shaded region exists that satisfying all three constraints if and only if the IC line is on the left hand of the interception point of the $H$-line and $L$-line (see the first panel of figure 4). When $\frac{t_{1}^{\prime}}{t_{0}^{\prime}}<\frac{t_{1}}{t_{0}}$, the $H$-line's horizontal

\footnotetext{
${ }^{13}$ The analysis so far has ignored other IC constraints on the agent, in particular, the one that prevents him from deviation to action plan 010. However, from the analysis of $O 0$, we learned that this constraint will not be binding so our analysis is justified.
} 

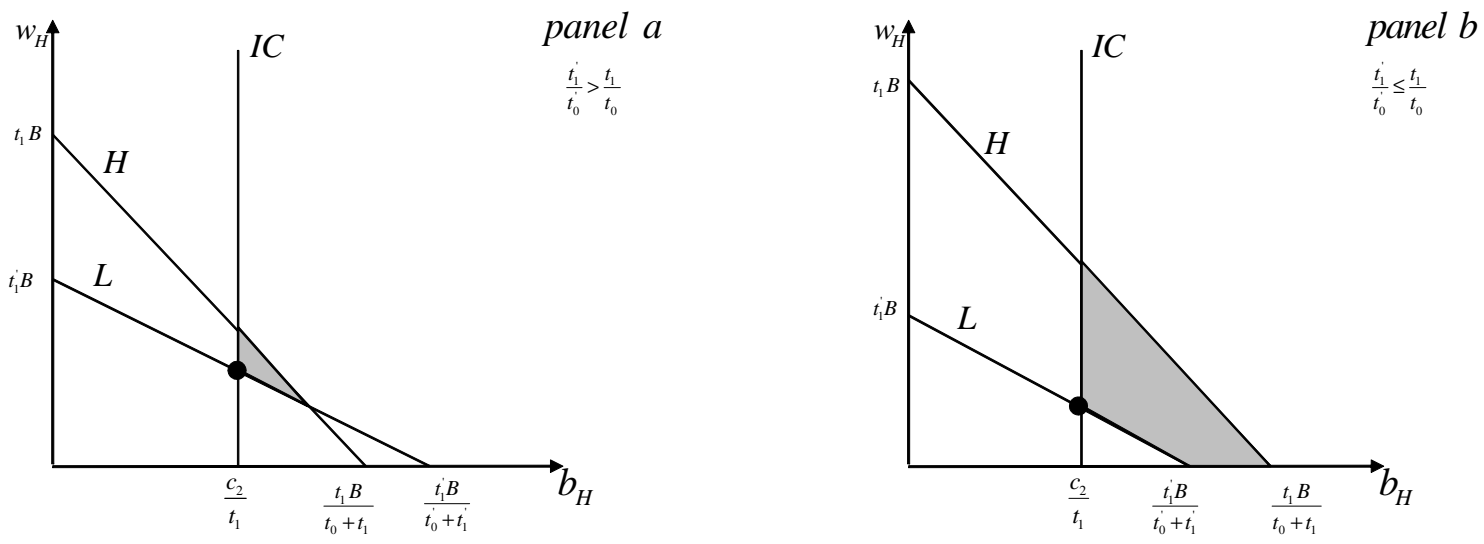

Figure 4: Two cases to consider when characterizing the optimal subjective contract

intercept is greater. A shaded region satisfying all three constraints exists if and only if The $I C$ line is on the left hand of that intercept (see the second panel of Figure 4). In both cases, a minimum $B$ is required for a solution to exist; and if it does there exists a shaded region and the optimal pair is the dotted point inside it. (Note that in the latter case, there is a chance that the neither $L$ nor $H$ is binding when $I C$ just stands strictly in between the two intercepts. ${ }^{14}$ )

It is interesting that $B$ appears in these diagrams, but not in the diagram for $S 1$ (Figure 3 ). The reason is that, under $S 1$, the same effort choice $\left(e_{2}=1\right)$ is called upon under different IPE outcomes and hence $B$ disappears in the principal's truth telling constraints (11) and (12). It is not the case under $S 0$.

Proposition 7 Consider the scenario where a subjective IPE is conducted.

\footnotetext{
${ }^{14}$ The intuition is that, under the effort-sorting scheme, the cheating motive of the $L$-type principal is reduced when $B$ is smaller. When the reduction is large enough, both truth telling constraints become binding even when $O 0$ is used.
} 
1. Suppose $\frac{t_{1}^{\prime}}{t_{0}^{\prime}}>\frac{t_{1}}{t_{0}}$. The action plan $\langle 1 ; 1,0\rangle$ is implementable if and only if $B \geq \frac{\left(t_{0}+t_{1}-t_{0}^{\prime}-t_{1}^{\prime}\right)}{\left(t_{1}-t_{1}^{\prime}\right)} \frac{c_{2}}{t_{1}}$.

2. Suppose $\frac{t_{1}^{\prime}}{t_{0}^{\prime}}<\frac{t_{1}}{t_{0}}$. The action plan $\langle 1 ; 1,0\rangle$ is implementable if and only if $B \geq \frac{\left(t_{0}+t_{1}\right)}{t_{1}} \frac{c_{2}}{t_{1}}$.

3. Suppose $\langle 1 ; 1,0\rangle$ is implementable. The optimal contract $S 1$ satisfies $b_{L}=w_{L}=0, b_{H}=\frac{c_{2}}{t_{1}}$; and $w_{H}=\max \left\{t_{1}^{\prime} B-\left(t_{0}^{\prime}+t_{1}^{\prime}\right) \frac{c_{2}}{t_{1}}, 0\right\}$.

Result 1 clarifies the conditions under which the effort-sorting scheme is implementable. Result 2 of Proposition 4 characterizes the optimal contract. Unlike an objective IPE, in which the optimal milestone contract satisfies $w_{H}=0$, a subjective IPE may dictate a positive $w_{H}$. The type- $H$ principal can distinguish herself from the type- $L$ principal by setting a relative low $b$ or a relative high $w$, because setting a reward $b$ is more costly for the type- $H$ than for the type- $L$, while setting a $w$ generates the same cost for the two types of principals. However, $b_{H}$ cannot be lowered, because it is the minimal requirement of bonus to induce high Stage-2 effort after revealing $H$. Hence, to deter cheating, $w_{H}$ might be more high-powered, resulting in greater costs than when an objective IPE is used.

This result sheds light on the finding by Bewley $(1995,1999)$ that a wage cut, or a low pay, will impact the worker's future productivity. This link is at odds with the traditional incentive theory, and it is usually attributed to "low morale." However, by stressing the "signalling" role of previous payments, our analysis identifies that a high wage rate paid out at the interim stage can better motivate the agent in the next stage, since it allows him to believe in success.

We next compare the implementation cost under $S 0$ with that under $T . S 0$ is preferred to $T$ if and only if $V^{T}-V^{S 0} \leq C^{T}-C^{S 0}$, where the LHS is the reduction of expected revenue and the RHS the reduction of expected cost. While the LHS is increasing in $B$, the RHS is decreasing in $B$ ( $C^{T}$ being independent of $B$ and $C^{S 0}$ and the RHS being decreasing in $B$ because of $w_{H}$, see proposition 7.3). Hence, there exists a critical value of $B$, denoted by $B^{S}$, such that $S 0$ generates a higher profit to the principal than $T$ if $B \leq B^{S}$. One can easily show that this $B^{S}$ is decreasing in $t_{1}^{\prime}$ (for the same reason that $B^{O}$ is decreasing in $t_{1}^{\prime}$ ). Here we summarize our result.

Proposition 8 There exists a cutoff $B^{S}$ such that S0 yields a higher (lower) level of profit to the principal than $T$ if $B<B^{S}\left(B>B^{S}\right)$. Moreover, this cutoff $B^{S}$ is increasing in $c_{2}, t_{0}^{\prime}$, and $t_{0}$ and decreasing in $t_{1}^{\prime}$. 


\subsection{The value of subjective IPE contracts}

Here we first discuss the optimal choice of contract when subjective IPE is available and then discuss the comparison of it with respect to the first best.

Optimal contract Figure 4 depicts the results. There is a downward slopping $B^{S}$, over which $T$ is preferred to $S 0$ and below which $T$ is less preferred to $S 0$. This $B^{S}$ plays a similar role as $B^{O}$ does in objective IPE. Another line $B^{\#}$ which is the minimum $B$ to ensure the implementability of action plan $\langle 1 ; 1,0\rangle$. According to Proposition 7.2, for $\frac{t_{1}^{\prime}}{t_{0}^{\prime}}<\frac{t_{1}}{t_{0}}$, this minimum $B$ is flat and equal to $\frac{\left(t_{0}+t_{1}\right)}{t_{1}} \frac{c_{2}}{t_{1}}$; fore $\frac{t_{1}^{\prime}}{t_{0}^{\prime}}>\frac{t_{1}}{t_{0}}$, the minimum $B$ is increasing in $t_{1}^{\prime}$. (It is interesting to note that these two minimum $B$ s agree with each other when $\frac{t_{1}^{\prime}}{t_{0}^{\prime}}=\frac{t_{1}}{t_{0}}$; we use $B^{\#}$ to denote this minimum $B$ in general.) Because $B^{\#}$ approaches infinity as $t_{1}^{\prime}$ approaches $t_{1}^{\prime}$, it must intersect with the downward slopping $B^{S}$ curve at some $t_{1}^{\prime}<t_{1}$. We denote by $\alpha$ the value of $t_{1}^{\prime}$ at the intersection point. Then for the range bounded by $B \#$ and $B^{S}$, over which the optimal contract is $S 0$, everywhere else the optimal contract is $T$. (Note that, for simplicity, we assume that $S 1$ is always preferred to $T^{\prime}$; in case there is possible that $S 1$ dominates $T$, then there will be a vertical strip of region with the $t_{1}^{\prime}=t_{1}$ line as its right hand, over which $S 1$ is the optimal choice.). ${ }^{15}$

Comparison with the first best The comparison of the optimal contract with the first best is similar to the one found in the last section with objective IPE. Basically, there will be three horizontal bands in a diagram like figure 4 . In the first band, the optimal contract implements the first best outcome; in the middle band, there is underinvestment in the optimal contract (implementing $\langle 1 ; 1,0\rangle$ rather than $\langle 1 ; 1,1\rangle)$; in the bottom band, the optimal contract implements the first best outcome (now equal to $\langle 1 ; 1,0\rangle$ ). However, because of the nature of subjective IPE, $\langle 1 ; 1,0\rangle$ may not be implemented even though the first best calls for it. This is the new element found in the comparison.

To conclude, despite our focus on Assumption 1, the following insights prevail: (i) for the principal, the subjectivity of the IPE imposes additional constraints on the use of milestone contracts, making the optimal subjective milestone contract less profitable than the optimal objective mile-

\footnotetext{
${ }^{15}$ Notice that there are three reasons why $S 0$ may be dominated by $T$ in region $F$. First, not choosing $e_{2}=1$ when $L$ signal is received is inefficient; second, the limited liability rents under IPE case are too large; third, the truth telling constraints on the principal impose not making effort under $L$-signal is indeed an unwise choice. While the two reasons also explain why $O 0$ may be dominated by $T$, the last reason is new here.
} 
stone contract; (ii) there still exist circumstances in which the optimal subjective milestone contract payoff-dominates the optimal traditional milestone contract. ${ }^{16}$

We have not studied IPE contracts for other action plans, it suffices to point out that our earlier result regarding $S 0$ that $w_{H} \geq 0$ and $w_{L}=0$ also holds for optimal milestone contracts for action plans $\langle 0 ; 1,0\rangle,\langle 1 ; 1,1\rangle$, and $\langle 0 ; 1,1\rangle$. The basic intuition is the same: to prevent the principal from lying about having observed signal $H$ when she had in fact observed signal $L$, who claims to have observed signal $H$ must be fined an amount equal to $w_{H}$.

\section{Discussions and extensions}

\subsection{More noise}

Thus far we assumed that the IPE evaluation is perfectly informative in the sense that, given the IPE outcome, the agent's private information about his own stage- 1 effort-whether $e_{1}=1$ or $e_{1}=0$-becomes irrelevant. It is realistic to assume that the IPE evaluation outcome is noisy; instead of knowing the quality of the interim product, the IPE is to receive a noisy signal of it. For instance, given a high quality of the interim product, there is a probability $q>0.5$ of receiving a good signal (and $1-q$ of receiving a bad signal); given a low quality of the interim product, there is a probability $1-q$ of receiving a good signal (and $q$ of receiving a bad signal). In preliminary work, we characterized the objective IPE contract that implements the action plan $\langle 1 ; 1,1\rangle$, finding out that the comparison of the likelihood ratios is still the main determinant of whether the contract is more profitable than the traditional contract. Because of this, we decided not to pursue it.

Another dose of realism can be added to the subjective IPE case. In the case of subjective IPE, we assume the IPE outcome is not observed by the agent (nor any correlated signal of it). We found that IPE is now not as valuable as when it is objective. In fact, it is realistic to assume that the agent, as well as the court, also observes some positively correlated signal of the IPE outcome. This scenario can be seen as an intermediate case between the objective IPE case and the subjective IPE case we have studied. We conjecture that, in this case, the IPE will be more useful than it is in the subjective IPE studied in this paper.

\footnotetext{
${ }^{16}$ In our analysis, we ignore mixed equilibrium in which a type of principal mixes when uttering message upon receiving IPE. The primary result for the omission is that we want to implement some deterministic action plan, whose implementation must involve complete separation of the two types of principal.
} 


\subsection{The agent can destroy evidence}

In the previous study of subjective IPE, we assume that the principal has private information on the interim feedback. However, it is possible that the agent observes the quality of the interim product and is able to destroy or manipulate the signal in his favor without the principal's detection. (We still assume that the quality itself is unchanged, however.) In this case, in order to implement an action plan, we need to make sure that the agent has no incentive to destroy evidence, i.e., to lie about the true quality of the interim product. Without loss of generality, we assume that if nil or any other message is claimed, the principal holds a belief that signal $L$ is detected by the agent in this off-equilibrium path. We denote by $A 1$ and $A 0$ the optimal contracts that implement action plans $\langle 1 ; 1,1\rangle$ and $\langle 1 ; 1,0\rangle$, respectively.

Consider action plan $\langle 1 ; 1,1\rangle$. The contract $O 1$ will not work because now the agent will lie that the quality is $L$ even though he has observed $H$, so as to earn a greater bonus. Suppose, besides the agent truth telling constraints, the only constraints that may be binding are constraints that prevent deviation in the second stage. In that case, one can easily show that the new optimal IPE contract entails that

$$
w_{H}=w_{L}=0 \text { and } b_{L}=b_{H}=\frac{c_{2}}{t_{1}^{\prime}} .
$$

This contract is the same as $O 1$ except that now $b_{H}$ is increased so that the agent will not lie when observing $H$. (Why $w_{L}$ is not used, as in the case of $S 1$ in which $w_{H}$ is used? intuition!!!) It is easy to show that the corresponding expected cost is higher than under $T$. Thus having IPE is actually harmful for the principal, so long as the action plan remains the same as under $T$. Thus far, we assume that the constraint that prevents first-stage deviation is not binding. If it does, that will further increase $b_{L}$ and $b_{H}$, and the domination of IPE contract by $T$ will be even more stark.

Next consider action plan $\langle 1 ; 1,0\rangle$. The contract $O 0$ will not work because now the agent will lie that the quality is indeed $H$ even though he has observed $L$, so as to earn $b_{H}$ with some probability. As a remedy, the optimal contract that implements $\langle 1 ; 1,0\rangle$ will entail a positive $w_{L}$ to prevent the agent from such lying. One can easily show that the contract satisfies: ${ }^{17}$

$$
w_{H}=b_{L}=0 ; b_{H}=\frac{c_{2}}{t_{1}} \text { and } w_{L}=\left(t_{0}^{\prime} / t_{1}\right) c_{2} .
$$

\footnotetext{
${ }^{17}$ It is interesting to notice a symmetry with respect to S0. Using O0 as a benchmark, under S0, wH is used as the main instructment to maintain the private information processor's truth telling incentive; under the optimal contract here, $\mathrm{wL}$ is used as the main instrument.
} 
(There also exists another solution similar to the solution of implementing $\langle 1 ; 1,1\rangle$. Instead of using a positive $w_{L}$, the principal could set $b_{L}=b_{H}=c_{2} / t_{1}$, while keeping $w_{H}=w_{L}=0$. One can show that this alternative contract corresponds to the same cost, and all the four truth telling constraints are satisfied.) Despite additional rent paid because of the agent's adverse selection problem, we can still identify a range of parameters (e.g., sufficiently low $B$ or $t_{1}^{\prime}$, or sufficiently high $c_{2}$ or $t_{1}$ ) to support the payoff dominance of the effort-sorting scheme over traditional contract. ${ }^{18}$

Proposition 9 1. Suppose $c_{1} / c_{2}$ is sufficiently small. The contract that implements $\langle 1 ; 1,1\rangle$ is characterized by (15). The agent cost under the IPE contract is greater than under $T$ (this does not rely on the assumption of $c_{1} / c_{2}$ being sufficiently large).

2. The contract that implements $\langle 1 ; 1,0\rangle$ is characterized by (16). There exist parameter values under which the IPE contract is more profitable than T.

It is useful to compare this proposition to early propositions about subjective IPE. The second result here is quite similar to those found earlier and does not require more elaboration. However, result 1 is not the case. Despite the apparent symmetry of this model with the subjective IPE, in which the principal has private information about the IPE outcome, we note two differences. The first is the characterization of the IPE contract. In the principal-private-information case, the separation instrument is wage, rather than bonus; in the agent-has-private-information case, the separation instrument is bonus, rather than wage. Second, there is also an asymmetry regarding the profitability of the IPE contracts that implement the same action plan. In the principal-privateinformation case, the IPE contract may still be profitable; in this agent-private-information case, the IPE will never be profitable.

\subsection{The agent has unknown ability}

In this paper, we assumed that there is only one type of agent and his productivity is commonly known. In reality, the agent may have different abilities and may not be certain about his exact productivity. To fix idea, consider the following modification to the model in section 3. Suppose there are two types of agent: $h$ (high) and $l$ (low), and it is commonly believed that the agent is of

\footnotetext{
${ }^{18}$ In the section on Subjective IPE contract, we found that implementation is an issue for action plan 110 . The reason is that in taht case B enters into the principal's truth telling constraints and so the aciton plan is not implementable for some range of B. In the current model, however, B does not enter into the agent's truth telling constraints and thus the problem does not arise.
} 
high type with probability of $\omega \in(0,1)$. Although both types having the same costs regarding their effort, the $h$-type agent is more productive than is the $l$-type, in the following sense. The $i$-type agent's stage- 1 effort increases the success probability of the interim product by probability $\theta_{i} r_{1}$; his stage- 2 effort increases the success probability of the final product by probability $\theta_{i} t_{1}\left(\theta_{i} t_{1}^{\prime}\right)$ when the interim product is high quality (low quality), where $\theta_{h}>\theta_{l}$. We continue to focus on the case where $c_{1} / c_{2}$ is sufficiently small and the optimal traditional contract is to implement action plan $\langle 1 ; 1\rangle$.

Suppose objective IPE is used. Upon a low quality through the IPE, the agent will now be less confident not only because the quality is low but also because the probability of his being low-typed is higher than previously thought and hence the success probability of stage- 2 effort is even slimmer. Thus, we conjecture that the main advantage of the IPE compared with the traditional contract is attributable more to the fact that it allows the principal to give different instruments to the agent conditional on the IPE outcome (to implement $\langle 1 ; 1,0\rangle$ ) than to the fact that it incentivizes with a lower cost the same effort in the stage 2 under both $H$ and $L$ IPE outcome (to implement $\langle 1 ; 1,1\rangle$ ).

Notice that given our assumptions, the principal will never be certain about the type of the agent even with the help of the IPE. However, we can envision scenarios in which the type of the agent is truly confirmed. For instance, suppose, with effort $e_{1}=1$, a $h$-type agent will always create an interim product of high quality, while a $l$-type agent will create an interim product of either quality with positive probability. In this case, the agent is certain to be of $l$-type once a $L$ IPE signal is observed. The bottom line is that IPE allows the principal to enhance her knowledge about the innate ability of the agent, and this provides an additional rationale why IPE may be contemplated. We leave this interesting issue for future studies.

\subsection{The opportunity of correction}

In reality, an early stage has resulted in a poor outcome may be repeated. The opportunity to redo the first task is an additional reason why conducing an IPE is beneficial to the principal. The simplest model to consider is the same model as in Section 2, except that, upon receiving the IPE outcome, the principal is allowed to ask the agent or somebody else to redo the Stage- 1 task. Assume that (i) a task can only be redone once; (ii) redoing a task incurs an extra cost $I$ to the principal due to the delay in project completion, additional materials, etc.; and (iii) redoing the first task is like starting it afresh, and previous experience is of no use in the second attempt. The final assumption 
has two implications. First, redoing the first task is valuable only if a $L$-signal is obtained. Second, in redoing the task, it is better to hire a new agent, for the following reason. If the original agent is retained, he would gain positive rent even though he failed to pass the milestone in the first attempt, hence the original moral hazard problem would be exacerbated.

The basic trade-off of imposing a correction is that subsequent to a poor IPE outcome, hiring a new agent to redo the previous task increases the probability of achieving the final success; but the likelihood of being fired makes it more costly to motivate the first agent, due to the worsened agency problem in Stage 1 as well as extra difficulty upon information disclosure. Thus, correction is favored by a larger $B$, but disfavored by a larger $c_{1} / c_{2}$ or $I$. One shall be able to find parameter values with which the optimal traditional contract (i) dominates any subjective milestone contract without the redo option and (ii) is dominated by some subjective milestone contracts with the redo option.

\subsection{Multiple agents}

Midterm reviews have also received significant attention in the recent literature on dynamic tournament. Some of these papers, including Yildirim (2005), Aoyagi (2008), Goltsman and Mukherjee (2009), Ederer (forthcoming), try to characterize the optimal strategy of interim information disclosure in the context of a two-stage tournament. Moreover, Gershkov and Perry (2009), while keeping a fixed disclosure policy, investigate the optimal aggregation and compensation rules in a tournament with or without midterm reviews. However, our model is built upon the standard principal-agent setting, instead of with multiple-agent competition. Another difference is that the contracting problem in our model goes hand in hand with information disclosure and morale formation.

\subsection{Extrinsic and Intrinsic Motivation}

The seminal work by Bénabou and Tirole (2003) studies the interplay between extrinsic incentive and intrinsic motivation. In that paper, the authors introduce notions such as self-confidence, trust, etc., into a specific class of principal agent model. As is in a standard principal agent model, the principal wants the agent to exert some effort to achieve some goal and the effort is costly. What is novel is that the agent does not know his own ability as much as the principal does and that the agent will receive some utility from achieving the goal (hence the name of intrinsic motivation). 
The Bayesian equilibrium is thus characterized by different types of principal giving different reward schemes to the agent, who infers that his ability is lower when he is promised a more powered reward. Benahue and Tirole are therefore able to relate notions, such as self-confidence, that are foreign to economics but popular in pyschology and education.

Notice that our model of objective IPE suggests that the "low ability" agent be given a stronger incentive, with the interpretation that "ability" corresponds to the quality outcome of the first stage of production. In this sense the result resembles that of Banahue and Tirole and in fact we have attributed the necessity of stronger incentive to non-economic notions such as the low morale or lack of self confidence of the agent. Notice that in our model there is no assymetric information at the outset - Benahue and Tirole assume there is - and the agent does not have an intrinsic motivation to complete the tasks-Benabue and Tirole assume there is. Our main point, therefore, is that notions such as low morale and self confidence have their justifiable places in even broader economic environments. ${ }^{19}$

\subsection{Empirical relevance}

To be filled in.

\section{Conclusions}

In this paper, we have studied a 2-stage principal-agent model with different specifications. We have shown that using an IPE may boost the principal's profit even though he did not plan to condition the continuation action plan on the IPE outcome. We have clarified the conditions under which the IPE is valuable. These conditions can be expressed in terms of effort complementarity, as well as the comparison between the likelihood ratios under different IPE outcomes. Albeit weaker, the domination of the IPE contract over the traditional contract still exists under subjective IPE where the principal's incentive to lie is a concern.

There are two directions that future studies can pursue. One is to weaken the assumption that the IPE outcome always reveals perfectly the quality of the interim product. Given the current modeling, the private information the agent possesses about whether he has exerted effort becomes

\footnotetext{
${ }^{19}$ The subjective IPE case also has a relevant connection with Benabue and Tirole (2003). There "ability" corresponds to quality outcome of the first step in the production process. Moreover, the "ability" is the principal's private information.
} 
irrelevant once the IPE outcome is known. Although with preliminary work we have shown that our main result is robust to a switch to noisy IPEs, we look forward to understanding the role of the degree of noisiness in IPEs in contracting. Another direction we look forward to is re-visiting the issue when efforts are substitutes, rather than complements. 


\section{References}

[1] Aoyagi, M., 2008. "Information Feedback in a Dynamic Tournament." Mimeo, The Institute of Social and Economic Research, Osaka University.

[2] Bewley, T. F., 1995. "A Depressed Labor Market as Explained by Participants." American Economic Review 85, 250-254.

[3] Bewley, T. F., 1999. Why Wages Don't Fall During a Recession, Harvard University Press.

[4] Bénabou, Robert, and Jean Tirole (2003) "Intrinsic and Extrinsic Motivation," Review of Economic Studies 70 (3), 489-520.

[5] Bolton, P., D. Scharfstein., 1998. "Corporate Finance, the Theory of the Firm, and Organizations." Journal of Economic Perspectives 12, 95-114.

[6] Calveras, A., Ganuza, J., Hauk, E., 2004. "Wild Bids. Gambling for Resurrection in Procurement Contracts." Journal of Regulatory Economics 26, pp. 41-68.

[7] Che, Y.-K., Yoo, S.-W., 2001. "Optimal Incentive for Teams." American Economic Review 91, pp. 525-541.

[8] Crémer, J., 1995. "Arm's Length Relationships." Quarterly Journal of Economics CX, pp. 275-295.

[9] Ederer, F., forthcoming. "Feedback and Motivation in Dynamic Tournaments." Journal of Economics and Management Strategy.

[10] Fuchs, W., 2007. "Contracting with Repeated Moral Hazard and Private Evaluations." American Economic Review 97, pp. 1432-1448.

[11] Gershkov, A., M. Perry., 2009. "Tournaments with Midterm Reviews." Games and Economic Behavior 66, pp. 162-190.

[12] Goltsman, M., Mukherjee, A., 2009. "Interim Performance Feedback in Multistage Tournaments: the Optimality of Partial Disclosure." Mimeo, University of Western Ontario and Michigan State University.

[13] Levin, J., 2003. "Relational Incentive Contracts." American Economic Review 93, pp. 835-857. 
[14] Lizzeri, A., M. Meyer., N. Persico., 2002. "The Incentive Effects of Interim Performance Evaluations." CARESS working paper \#02-09.

[15] MacLeod, W.B., 2003. "Optimal Contracting with Subjective Evaluation." American Economic Review 93, pp. 216-240.

[16] Manso, G., 2010. "Motivating Innovation." Mimeo, MIT Sloan School of Management.

[17] Meredith, J., S. Mantel., 1995. Project Management: a Managerial Approach. 3rd edition, New York: John Wiley \& Sons, Inc, pp. 125-126 \& 446.

[18] Murphy, K., 1992. "Performance Measurement and Appraisal: Motivating Managers to Identify and Reward Performance." in William J. Burns, Jr., eds., Performance Measurement, Evaluation, and Incentives. Boston: Harvard Business School Press.

[19] Prendergast, C., 1999. "The Provision of Incentives in Firms." Journal of Economic Literature 37, pp. 7-63.

[20] Raith, M., 2009. "Optimal Incentives and the Time Dimension of Performance Measurement." Mimeo, University of Rochester.

[21] Ray, K., 2007a. "Performance Evaluations and Efficient Sorting." Journal of Accounting Research 45 , pp. 839-882.

[22] Ray, K., 2007b. "The Retention Effect of Withholding Performance Information." The Accounting Review 82, pp. 389-425.

[23] Reid, A., 1999. Project Management: Getting It Right. Cambridge: Woodhead Publishing Ltd.

[24] Roberts, J., 2004. The Modern Firm: Organizational Design for Performance and Growth. New York: Oxford University Press.

[25] Scharfstein, D., J. Stein., 2000. "The Dark Side of the Internal Capital Markets: Divisional Rent Seeking and Inefficient Investments." Journal of Finance 55, pp. 2537-2564.

[26] Schmitz, P.W., 2005. "Allocating Control in Agency Problems with Limited Liability and Sequential Hidden Actions." RAND Journal of Economics 36, pp. 318-336. 
[27] Yildirim, H., 2005. "Contests with Multiple Rounds." Games and Economic Behavior 51, pp. 213-227.

[28] Zhao, R., 2009. "Productive Low Morale." Economics Letters 103, pp. 18-22. 


\section{Appendix A: Proofs}

\section{Proof of Lemma 1}

Proof. Suppose the principal wants to implement the action plan $\langle x ; y, z\rangle$. No matter what $x, y$ and $z$ are, $w_{L}$ should be zero in the cost-minimized contract, since it helps nothing except for diluting the incentive in Stage 1. Then we consider the choice of $w_{H}$. It incentivizes the Stage-1 effort, so it would be useful only if $x=1$. However, we claim that $w_{H}$ cannot be a more cost-efficient instrument than $b_{H}$ is for inducing $e_{1}=1$.

Consider the case where $x=1$. If the principal increases one unit of $w_{H}$, the cost is $\left(r_{0}+r_{1}\right)$, while the Stage-1 IC constraints would be relaxed by the amount of $r_{1}$. However, if the principal increases one unit of $b_{H}$, the cost is $\left(r_{0}+r_{1}\right)\left(t_{0}+y t_{1}\right)$; the IC constraints for preventing deviation to $\langle 0 ; y, z\rangle$ would be relaxed by $r_{1}\left(t_{0}+y t_{1}\right)$, while the IC constraints for preventing deviation to $\langle 0 ; 0,0\rangle$ is relaxed by the amount of $\left[r_{0} t_{1}+r_{1}\left(t_{0}+t_{1}\right)\right]$. So by comparing the cost-benefit ratio, we found that $b_{H}$ is at least as cost-efficient as $w_{H}$ for relaxing relevant IC constraints.

\section{The non-equivalence between $w_{H}$ and $b_{H}$ when IPE outcome is noisy}

Here we show why in general Lemma 1 does not hold when the IPE outcome is noisy. Consider the following changes to the model presented in Section 2: Given that the intermediate good is of good (bad) quality, the IPE signal is $H$ with probability $q(1-q)$ and $L$ with probability $1-q(q)$, where $q>1 / 2$. Then the expected cost of implementing action plan $\langle 1 ; 1,1\rangle$ equals

$$
\begin{aligned}
C= & \left(r_{0}+r_{1}\right)\left[q\left(w_{H}+\left(t_{0}+t_{1}\right) b_{H}\right)+(1-q)\left(w_{L}+\left(t_{0}+t_{1}\right) b_{L}\right)\right] \\
& +\left(1-r_{0}-r_{1}\right)\left[(1-q)\left(w_{H}+\left(t_{0}^{\prime}+t_{1}^{\prime}\right) b_{H}\right)+q\left(w_{L}+\left(t_{0}^{\prime}+t_{1}^{\prime}\right) b_{L}\right)\right] .
\end{aligned}
$$

Therefore we reckon that

$$
\begin{aligned}
\frac{d C}{d w_{H}} & =(2 q-1)\left(r_{0}+r_{1}\right)+(1-q)>0 \\
\frac{d C}{d b_{H}} & =(r 0+r 1)\left(q(t 0+t 1)-(1-q)\left(t 0^{\prime}+t 1^{\prime}\right)\right)+\left(r 0^{\prime}+r 1^{\prime}\right)(1-q)>0 .
\end{aligned}
$$


To show that in general it is using $b_{H}$ and using $w_{H}$ are different, here we consider the IC constraint that prevents deviation to action plan $\langle 0 ; 1,1\rangle$ is as follows:

$$
\begin{aligned}
& \left(r_{0}+r_{1}\right)\left[q\left(w_{H}+\left(t_{0}+t_{1}\right) b_{H}\right)+(1-q)\left(w_{L}+\left(t_{0}+t_{1}\right) b_{L}\right)\right] \\
& +\left(1-r_{0}-r_{1}\right)\left[(1-q)\left(w_{H}+\left(t_{0}^{\prime}+t_{1}^{\prime}\right) b_{H}\right)+q\left(w_{L}+\left(t_{0}^{\prime}+t_{1}^{\prime}\right) b_{L}\right)\right]-c_{1}-c_{2} \\
> & r_{0}\left[q\left(w_{H}+\left(t_{0}+t_{1}\right) b_{H}\right)+(1-q)\left(w_{L}+\left(t_{0}+t_{1}\right) b_{L}\right)\right] \\
& +\left(1-r_{0}\right)\left[(1-q)\left(w_{H}+\left(t_{0}^{\prime}+t_{1}^{\prime}\right) b_{H}\right)+q\left(w_{L}+\left(t_{0}^{\prime}+t_{1}^{\prime}\right) b_{L}\right)\right]-c_{2}
\end{aligned}
$$

Rearranging, we have

$$
\begin{aligned}
& r_{1}\left[q\left(w_{H}+\left(t_{0}+t_{1}\right) b_{H}\right)-(1-q)\left(w_{H}+\left(t_{0}^{\prime}+t_{1}^{\prime}\right) b_{H}\right)\right]-c_{1} \\
> & r_{1}\left[+q\left(w_{L}+\left(t_{0}^{\prime}+t_{1}^{\prime}\right) b_{L}\right)-(1-q)\left(w_{L}+\left(t_{0}+t_{1}\right) b_{L}\right)\right],
\end{aligned}
$$

where $w_{H}$ and $b_{H}$ appear only in the LHS. Denote by $d I C / d w_{H}$ and $d I C / d b_{H}$ the amount the IC constraint is relaxed by one unit increase of $w_{H}$ and of $b_{H}$, respectively. It is straightforward to show that

$$
\frac{d I C}{d w_{H}}=r_{1}(2 q-1)>0
$$

and

$$
\frac{d I C}{d b_{H}}=\frac{2 q-1}{q\left(t_{0}+t_{1}\right)-(1-q)\left(t_{0}^{\prime}+t_{1}^{\prime}\right)}>0
$$

The cost-benefit ratio of using $w_{H}$ is

$$
\frac{\frac{d C}{d w_{H}}}{\frac{d I C}{d w_{H}}}=\frac{r_{0}+r_{1}}{r_{1}}+\frac{1}{2 q-1} \frac{1-q}{r_{1}}
$$

and the cost-benefit ratio of using $b_{H}$ is

$$
\begin{aligned}
\frac{\frac{d C}{d b_{H}}}{\frac{d I C}{d b_{H}}} & =\frac{r_{0}+r_{1}}{r_{1}}+\frac{t_{0}^{\prime}+t_{1}^{\prime}}{q\left(t_{0}+t_{1}\right)-(1-q)\left(t_{0}^{\prime}+t_{1}^{\prime}\right)} \frac{1-q}{r_{1}} \\
& =\frac{r_{0}+r_{1}}{r_{1}}+\frac{1}{q\left(\frac{t_{0}+t_{1}}{\left(t_{0}^{\prime}+t_{1}^{\prime}\right)}+1\right)-1} \frac{1-q}{r_{1}} \leq \frac{\frac{d C}{d w_{H}}}{\frac{d I C}{d w_{H}}}
\end{aligned}
$$


where the equality holds if and only if $q=1$. Therefore, for $q \in(0.5,1)$, it is strictly better to use $b_{H}$ instead of $w_{H}$ to provide incentive for the agent in the first stage.

\section{Proof of Proposition 1 (characterization of O1)}

Proof. Part 1. The cost minimization problem for inducing action plan $\langle 1 ; 1,1\rangle$ is as follows:

$$
\begin{aligned}
\min _{w_{H}, w_{L}, b_{H}, b_{L}} C^{O 1}= & \left(r_{0}+r_{1}\right) w_{H}+\left(1-r_{0}-r_{1}\right) w_{L} \\
& +\left(r_{0}+r_{1}\right)\left(t_{0}+t_{1}\right) b_{H}+\left(1-r_{0}-r_{1}\right)\left(t_{0}^{\prime}+t_{1}^{\prime}\right) b_{L},
\end{aligned}
$$

subject to the following IC constraints: the IC constraints that prevent deviation to $\langle 1 ; 0,1\rangle$ and $\langle 1 ; 1,0\rangle$, respectively (i.e., (5) and (6); the IC constraint that prevents deviation to $\langle 0 ; 1,1\rangle$ (i.e., (7)); and the IC constraint that prevents deviation to $\langle 0 ; 0,0\rangle$ :

$$
\begin{gathered}
{\left[r_{0} t_{1}+r_{1}\left(t_{0}+t_{1}\right)\right] b_{H}+\left[\left(1-r_{0}\right) t_{1}^{\prime}-r_{1}\left(t_{0}^{\prime}+t_{1}^{\prime}\right)\right] b_{L}} \\
+r_{1}\left(w_{H}-w_{L}\right) \geq c_{1}+c_{2} ;
\end{gathered}
$$

The claim that $w_{H}^{O 1}=w_{L}^{O 1}=0$ is obtained from Lemma 1. To find out the bonuses, let us define

$$
\gamma \equiv \frac{r_{1} t_{0}}{t_{1}}-\frac{r_{1} t_{0}^{\prime}}{t_{1}^{\prime}}
$$

We can verify that that (i) If $c_{1}<\gamma c_{2}$, only (5) and (6) are binding and others constraints are non-binding; as a result, $b_{H}$ and $b_{L}$ satisfy $b_{H}^{O 1}=\frac{c_{2}}{t_{1}}, b_{L}^{O 1}=\frac{c_{2}}{t_{1}^{\prime}}$.(ii) If $c_{1} \geq \gamma c_{2}$, only (6) and (7) are binding and others are non-binding; as a result $b_{H}$ and $b_{L}$ satisfy

$$
\begin{aligned}
b_{H}^{O 1} & =\frac{c_{1}}{r_{1}\left(t_{0}+t_{1}\right)}+\frac{\left(t_{0}^{\prime}+t_{1}^{\prime}\right) c_{2}}{\left(t_{0}+t_{1}\right) t_{1}^{\prime}}, \\
b_{L}^{O 1} & =\frac{c_{2}}{t_{1}^{\prime}}
\end{aligned}
$$

(Note that in any case, (17) is a non-binding constraints. It can be explained by one-deviation property.) (iii) $c_{1}<\gamma c_{2}$ if and only if

$$
\frac{c_{2}}{t_{1}}>\frac{c_{1}}{r_{1}\left(t_{0}+t_{1}\right)}+\frac{\left(t_{0}^{\prime}+t_{1}^{\prime}\right) c_{2}}{\left(t_{0}+t_{1}\right) t_{1}^{\prime}}
$$


Hence, (i) to (iii) establish the claim that $b_{H}^{O 1}=\max \left\{\frac{c_{2}}{t_{1}}, \frac{c_{1}}{r_{1}\left(t_{0}+t_{1}\right)}+\frac{\left(t_{0}^{\prime}+t_{1}^{\prime}\right) c_{2}}{\left(t_{0}+t_{1}\right) t_{1}^{\prime}}\right\}$ and $b_{L}^{O 1}=\frac{c_{2}}{t_{1}^{\prime}}$.

\section{Proof of Proposition 2}

Proof. Part 2. We now compare implementation costs. To simplify notation, we define

$$
\begin{aligned}
R & \equiv\left(r_{0}+r_{1}\right) ; \\
\alpha_{H} & \equiv R\left(t_{0}+t_{1}\right), \alpha_{L} \equiv(1-R)\left(t_{0}^{\prime}+t_{1}^{\prime}\right) ;
\end{aligned}
$$

then using (3) and (4) we reckon that $C^{T}=\left(\alpha_{H}+\alpha_{L}\right) b^{T}$. Suppose $c_{1}<\gamma c_{2}$ where $\gamma \equiv \frac{r_{1} t_{0}}{t_{1}}-\frac{r_{1} t_{0}^{\prime}}{t_{1}^{\prime}}$. Then $b_{H}^{O 1}=c_{2} / t_{1}$ and, with some manipulation, we have

$$
C^{O 1}=\left(\alpha_{H} \beta_{H}+\alpha_{L} \beta_{L}\right) b^{T}
$$

where

$$
\beta_{H} \equiv \frac{\left[R t_{1}+(1-R) t_{1}^{\prime}\right]}{t_{1}}, \beta_{L} \equiv \frac{\left[R t_{1}+(1-R) t_{1}^{\prime}\right]}{t_{1}^{\prime}}
$$

Hence

$$
\begin{aligned}
C^{T}-C^{O 1} & =\left(\alpha_{H}+\alpha_{L}\right) b^{T}-\left(\alpha_{H} \beta_{H}+\alpha_{L} \beta_{L}\right) b^{T} \\
& =\left[\alpha_{H}\left(1-\beta_{H}\right)+\alpha_{L}\left(1-\beta_{L}\right)\right] b^{T}
\end{aligned}
$$

Substituting the following into the expression

$$
1-\beta_{H}=1-\frac{\left[R t_{1}+(1-R) t_{1}^{\prime}\right]}{t_{1}}=\frac{(1-R)\left(t_{1}-t_{1}^{\prime}\right)}{t_{1}}
$$

and

$$
1-\beta_{L}=1-\frac{\left[R t_{1}+(1-R) t_{1}^{\prime}\right]}{t_{1}^{\prime}}=-\frac{R\left(t_{1}-t_{1}^{\prime}\right)}{t_{1}^{\prime}}
$$


and with some manipulation, we obtain

$$
\begin{aligned}
C^{T}-C^{O 1} & =\left(R(1-R)\left(t_{1}-t_{1}^{\prime}\right) \frac{\left(t_{0}+t_{1}\right)}{t_{1}}-R(1-R)\left(t_{1}-t_{1}^{\prime}\right) \frac{\left(t_{0}^{\prime}+t_{1}^{\prime}\right)}{t_{1}^{\prime}}\right) b^{T} \\
& =\left(R(1-R)\left(t_{1}-t_{1}^{\prime}\right)\left(\frac{t_{0}+t_{1}}{t_{1}}-\frac{t_{0}^{\prime}+t_{1}^{\prime}}{t_{1}^{\prime}}\right)\right) b^{T} \\
& =R(1-R)\left(t_{1}-t_{1}^{\prime}\right)\left(\frac{t_{0}}{t_{1}}-\frac{t_{0}^{\prime}}{t_{1}^{\prime}}\right) b^{T} .
\end{aligned}
$$

Since $b^{T}>0$ and $t_{1}>t_{1}^{\prime}, C^{T}-C^{O 1} \geq 0$ if and only if

$$
\frac{t_{0}}{t_{1}} \geq \frac{t_{0}^{\prime}}{t_{1}^{\prime}}
$$

Next, we consider the case where $c_{1} \geq \gamma c_{2}$. In this case,

$$
b_{H}^{O 1}=\frac{c_{1}}{r_{1}\left(t_{0}+t_{1}\right)}+\frac{\left(t_{0}^{\prime}+t_{1}^{\prime}\right) c_{2}}{\left(t_{0}+t_{1}\right) t_{1}^{\prime}}>\frac{c_{2}}{t_{1}}
$$

and the above calculation underestimates the true $C^{O 1}$. Hence, a fortiori, it must hold true that $C^{O 1}>C^{T}$ when $\frac{t_{0}}{t_{1}}<\frac{t_{0}^{\prime}}{t_{1}^{\prime}}$.

To conclude, given $\frac{t_{0}}{t_{1}}<\frac{t_{0}^{\prime}}{t_{1}^{\prime}}, C^{T}<C^{O 1}$. Given $\frac{t_{0}}{t_{1}}<\frac{t_{0}^{\prime}}{t_{1}^{\prime}}$, for sufficiently small $c_{1} / c_{2}$, we have $C^{T}>C^{O 1}$. The proof is thus complete.

\section{Proof of Proposition 3 (Characterization of O0)}

Proof. When implementing $\langle 1 ; 1,0\rangle$, the principal's cost-minimization problem is as follows.

$$
\begin{aligned}
\min C^{O 0} & =\left(r_{0}+r_{1}\right)\left(t_{0}+t_{1}\right) b_{H}+\left(1-r_{0}-r_{1}\right) t_{0}^{\prime} b_{L} \\
& +\left(r_{0}+r_{1}\right) w_{H}+\left(1-r_{0}-r_{1}\right) w_{L},
\end{aligned}
$$

subject to the following IC constraints.

The IC constraint that prevents deviation to $\langle 1 ; 0,0\rangle$ is (??). The IC constraint that prevents 
deviation to $\langle 0 ; 0,0\rangle$ is

$$
\begin{gathered}
{\left[\left(r_{0}+r_{1}\right) t_{1}+r_{1} t_{0}\right] b_{H}-r_{1} t_{0}^{\prime} b_{L}} \\
+r_{1}\left(w_{H}-w_{L}\right) \geq c_{1}+\left(r_{0}+r_{1}\right) c_{2} .
\end{gathered}
$$

The IC constraint that prevents deviation to $\langle 0 ; 1,0\rangle$ is

$$
r_{1}\left(t_{0}+t_{1}\right) b_{H}-r_{1} t_{0}^{\prime} b_{L}+r_{1}\left(w_{H}-w_{L}\right) \geq c_{1}+r_{1} c_{2} .
$$

First, let note that a positive $b_{L}$ or $w_{L}$ only dilutes incentive in Stage 1, so both of them should be zero. Moreover, according to Lemma $1, w_{H}$ is less cost-efficient than $b_{H}$ in relaxing the Stage-1 IC constraint. In the optimal $O 0$ contract, only $b_{H}$ is positive, while others equal to zero. Second, given Assumption 1, only (??) would be binding among all the IC constraints, so $b_{H}^{O 0}=\frac{c_{2}}{t_{1}}$.

\section{Proof of Proposition 4}

Proof. We reckon that the implementation cost of $O 0$ is

$$
C^{O 0}=\frac{\left(r_{0}+r_{1}\right)\left(t_{0}+t_{1}\right) c_{2}}{t_{1}}
$$

Implementing $O 0$ is more profitable than implementing $T$, i.e.,

$$
V^{O 0}-C^{O 0}>V^{T}-C^{T}
$$

if and only if

$$
\begin{aligned}
& \left(1-r_{0}-r_{1}\right) t_{1}^{\prime} B<\left[\frac{\left(r_{0}+r_{1}\right) t_{0}+\left(1-r_{0}-r_{1}\right) t_{0}^{\prime}}{\left(r_{0}+r_{1}\right) t_{1}+\left(1-r_{0}-r_{1}\right) t_{1}^{\prime}} c_{2}\right. \\
& \left.-\frac{\left(r_{0}+r_{1}\right) t_{0}}{t_{1}} c_{2}\right]+\left(1-r_{0}-r_{1}\right) c_{2} .
\end{aligned}
$$

or $B<B^{O}$, where

$$
B^{O} \equiv \frac{1}{\left(1-r_{0}-r_{1}\right) t_{1}^{\prime}}\left(\frac{\left(r_{0}+r_{1}\right) t_{0}+\left(1-r_{0}-r_{1}\right) t_{0}^{\prime}}{\left(r_{0}+r_{1}\right) t_{1}+\left(1-r_{0}-r_{1}\right) t_{1}^{\prime}} c_{2}-\frac{\left(r_{0}+r_{1}\right) t_{0}}{t_{1}} c_{2}\right)+\frac{c_{2}}{t_{1}^{\prime}}
$$


It is straightforward to show that $B^{O}$ is increasing in $c_{2}, t_{0}^{\prime}, t_{0}$, but is decreasing in $t_{1}^{\prime}$.

\section{proof of Proposition 6 (characterization of subjective IPE, S1)}

Proof. For $c_{1} / c_{2}$ sufficiently small, we reckon that the implementation cost of $S 1$ is

$$
C^{S 1}=\left(r_{0}+r_{1}\right)\left(t_{0}+t_{1}-t_{0}^{\prime}-t_{1}^{\prime}\right) \frac{c_{2}}{t_{1}}+\left(t_{0}^{\prime}+t_{1}^{\prime}\right) \frac{c_{2}}{t_{1}^{\prime}} .
$$

Differentiating it with respect to $t_{1}^{\prime}$, we have

$$
\frac{\partial C^{S 1}}{\partial t_{1}^{\prime}}=-\left(r_{0}+r_{1}\right) \frac{c_{2}}{t_{1}}-\frac{t_{0}^{\prime}}{\left(t_{1}^{\prime}\right)^{2}} c_{2}
$$

Differentiating $C^{T}$, (4), with respect to $t_{1}^{\prime}$, we have

$$
\frac{\partial C^{T}}{\partial t_{1}^{\prime}}=-\left(1-r_{0}-r_{1}\right) \frac{\left[\left(r_{0}+r_{1}\right) t_{0}+\left(1-r_{0}-r_{1}\right) t_{0}^{\prime}\right]}{\left[\left(r_{0}+r_{1}\right) t_{1}+\left(1-r_{0}-r_{1}\right) t_{1}^{\prime}\right]^{2}} c_{2}
$$

Focusing at the point where $t_{1}^{\prime}=t_{1}$, we have

$$
\left.\frac{\partial C^{S 1}}{\partial t_{1}^{\prime}}\right|_{t_{1}^{\prime}=t_{1}}=-\left(r_{0}+r_{1}+\frac{t_{0}^{\prime}}{t_{1}}\right) \frac{c_{2}}{t_{1}}<0
$$

and

$$
\left.\frac{\partial C^{T}}{\partial t_{1}^{\prime}}\right|_{t_{1}^{\prime}=t_{1}}=-\left(1-r_{0}-r_{1}\right) \frac{\left[\left(r_{0}+r_{1}\right) t_{0}+\left(1-r_{0}-r_{1}\right) t_{0}^{\prime}\right]}{t_{1}^{2}} c_{2}<0 .
$$

It is easy to show that

$$
\left.\frac{\partial C^{S 1}}{\partial t_{1}^{\prime}}\right|_{t_{1}^{\prime}=t_{1}}>\left.\frac{\partial C^{S 1}}{\partial t_{1}^{\prime}}\right|_{t_{1}^{\prime}=t_{1}}
$$

if and only if

$$
\left(1-r_{0}-r_{1}\right)\left(t_{0}-t_{0}^{\prime}\right)>t_{1}+t_{0}^{\prime}
$$

Notice that if $t_{1}^{\prime}=\frac{t_{0}^{\prime}}{t_{0}} t_{1}, C^{S 1} \geq C^{O 1}=C^{T}$ and if $t_{1}^{\prime}=t_{1}, C^{S 1}=C^{T}$. Altogether, this suggests that there exists $\alpha \in\left(\left(t_{0}^{\prime} / t_{0}\right) t_{1}, t_{1}\right)$ such that for all $t_{1}^{\prime} \in\left(\alpha, t_{1}\right)$, the implementation cost under $S 1$ is lower than under $T$. This completes the proof. (Notice that the condition (20) is very restrictive but is not impossible. For example, when $r_{0}+r_{1}=0.4, t_{0}=0.5, t_{0} \prime \doteq 0$, then the $t_{1}$ that satisfies 
this condition can be as high as 0.3 . If $t_{1}^{\prime}$ is close to 0.3 as well, then $t_{1}^{\prime} / t_{0}$ is extremely high. But this is precisely what makes IPE useful.)

\section{Proof of Proposition 7 (characterization of subjective IPE)}

Proof. To characterize the optimal contract $S 0$, let note that the cost-minimization problem is the same as described in the proof of Proposition 2, except that (13) and (14) are added. Then we claim that the choice of $w_{L}$ and $b_{L}$ should be zero, because they strain the IC constraints but cannot relax the truth-telling constraints.

Using the graphical method described in the main text, we find that

(1) When $\frac{t_{1}^{\prime}}{t_{0}^{\prime}}>\frac{t_{1}}{t_{0}}$ (refer to panel a of Figure 4), the "IC" line should be in the left of the crossing point of two truth-telling constraints. The corresponding condition is

$$
B>\frac{\left(t_{0}+t_{1}-t_{0}^{\prime}-t_{1}^{\prime}\right)}{\left(t_{1}-t_{1}^{\prime}\right)} \frac{c_{2}}{t_{1}} .
$$

(2)When $\frac{t_{1}^{\prime}}{t_{0}^{\prime}}<\frac{t_{1}}{t_{0}}$ (refer to panel b of Figure 4), , the "IC" line should be in the left of the intercept of the " $H$ " line with the horizontal axis. The corresponding condition is

$$
B>\frac{\left(t_{0}+t_{1}\right)}{t_{1}} \frac{c_{2}}{t_{1}}
$$

So the two necessary conditions of implementation is summarized in Part (i) of the Proposition. The remaining work is to characterize the cost-minimal contract among all the feasible solutions. The optimal choice is depicted by the dark point, and the choices of $b_{H}$ and $w_{H}$ are presented in Part (3).

\section{Proof of Proposition 8 (value of subjective IPE)}

Proof. We compare $S 0$ with $T$. $S 0$ payoff-dominates $T$ if

$$
V^{T}-V^{S 0} \leq C^{T}-C^{S 0}
$$


In case that $w_{H}>0,(21)$ is equivalent to

$$
\begin{aligned}
& \left(1-r_{0}-r_{1}\right) t_{1}^{\prime} B \leq\left[\frac{\left(r_{0}+r_{1}\right) t_{0}+\left(1-r_{0}-r_{1}\right) t_{0}^{\prime}}{\left(r_{0}+r_{1}\right) t_{1}+\left(1-r_{0}-r_{1}\right) t_{1}^{\prime}} c_{2}-\left(r_{0}+r_{1}\right) \frac{t_{0}}{t_{1}} c_{2}\right] \\
& +\left(1-r_{0}-r_{1}\right) c_{2}-\left(r_{0}+r_{1}\right)\left[t_{1}^{\prime} B-\left(t_{0}^{\prime}+t_{1}^{\prime}\right) \frac{c_{2}}{t_{1}}\right]
\end{aligned}
$$

which is rearranged to

$$
\begin{aligned}
B \leq & B^{\prime} \equiv \frac{1}{t_{1}^{\prime}}\left[\frac{\left(r_{0}+r_{1}\right) t_{0}+\left(1-r_{0}-r_{1}\right) t_{0}^{\prime}}{\left(r_{0}+r_{1}\right) t_{1}+\left(1-r_{0}-r_{1}\right) t_{1}^{\prime}}-\left(r_{0}+r_{1}\right) \frac{t_{0}}{t_{1}}\right] c_{2} \\
& +\frac{1}{t_{1}^{\prime}}\left(1-r_{0}-r_{1}\right) c_{2}+\left(r_{0}+r_{1}\right) \frac{\left(t_{0}^{\prime}+t_{1}^{\prime}\right)}{t_{1}^{\prime}} \frac{c_{2}}{t_{1}}
\end{aligned}
$$

On the other hand, in case that $w_{H}=0,(21)$ is equivalent to $B \leq B^{O}$. Thus, $B^{S}$ is defined as follows.

$$
B^{S}=\min \left\{B^{\prime}, B^{O}\right\}
$$

It is clear that $S 0$ is implementable and yields a higher profit than $T$, if $B^{\#} \leq B \leq B^{S}$. The remaining work is to prove that such range of $B$ does exists.

Claim 1 There exists $\alpha \geq \frac{t_{0}^{\prime}}{t_{0}} t_{1}$ such that if $t_{1}^{\prime}<\alpha$, the range of $\left[B^{\#}, B^{S}\right]$ exists; if $t_{1}^{\prime} \geq \alpha$, no range of $B$ exists to support the optimality of choosing the effort sorting scheme.

First, if $t_{1}^{\prime}=\frac{t_{0}^{\prime}}{t_{0}} t_{1}$

$$
B^{S} \geq B^{O}=\frac{1}{t_{1}^{\prime}} \frac{\left(t_{0}+t_{1}\right)}{t_{1}} c_{2} \geq \frac{1}{t_{1}} \frac{\left(t_{0}+t_{1}\right)}{t_{1}} c_{2}=B^{\#}
$$

Second, if $t_{1}^{\prime} \rightarrow t_{1}$,

$$
B^{\#}=\infty>B^{S}
$$

Third, we know that $B^{S}$ is decreasing in $t_{1}^{\prime}$, while $B^{\#}$ is weakly increasing in $t_{1}^{\prime}$. Thus, according to intermediate value theorem, we could find a $\alpha \geq \frac{t_{0}^{\prime}}{t_{0}} t_{1}$ satisfying the claim.

Thus, in combination with Claim 1, the proof is completed.

\section{Proof of Proposition 9 (the agent can destroy evidence)}

Proof. Consider contract $A 1$, which is to implement action plan $\langle 1 ; 1,1\rangle$. The agent's truth telling 
constraints are the following. The first constraint is no incentive to misreporting $L$ when actually observing $H$.

$$
\left(t_{0}+t_{1}\right) b_{H}-c_{2}+w_{H} \geq \max \left\{\left(t_{0}+t_{1}\right) b_{L}-c_{2}+w_{L}, t_{0} b_{L}+w_{L}\right\},
$$

where the LHS is the payoff when he truthfully reports and exerts effort $e_{2}=1$, and the RHS is the maximum payoff between two possible deviations: reporting $L$ and exerting effort versus reporting $L$ and not exerting effort.

The second IC constraint is no incentive to misreporting $H$ when actually observing $L$.

$$
\left(t_{0}^{\prime}+t_{1}^{\prime}\right) b_{L}-c_{2}+w_{L} \geq \max \left\{\left(t_{0}^{\prime}+t_{1}^{\prime}\right) b_{H}-c_{2}+w_{H}, t_{0}^{\prime} b_{H}+w_{H}\right\}
$$

where the LHS is the payoff when he truthfully reports and exerts effort according to the plan, and the RHS is the maximum payoff between two possible deviations: reporting $H$ and exerting effort versus reporting $H$ and not exerting effort.

Note that the RHS of (22) describes the payoff when the agent claims $L$ but still exerts high effort in Stage 2. However, when observing $L$, the agent would not cheat by claiming $H$. Now consider the milestone contract that implements $\langle 1 ; 1,0\rangle$. When observing $H$, the agent would not cheat by claiming $L$, if

$$
\left(t_{0}+t_{1}\right) b_{H}-c_{2}+w_{H} \geq t_{0} b_{L}+w_{L}
$$

when observing $L$, the agent would not cheat by claiming $H$, if

$$
\begin{aligned}
& t_{0}^{\prime} b_{L}+w_{L} \geq\left(t_{0}^{\prime}+t_{1}^{\prime}\right) b_{H}-c_{2}+w_{H} . \\
& \left(t_{0}+t_{1}\right) b_{H}-c_{2}+w_{H} \geq t_{0} b_{L}+w_{L} .
\end{aligned}
$$

We claim that as long as $b_{L}=\frac{c_{2}}{t_{1}^{\prime}},(26)$ would not be binding, because

$$
\begin{aligned}
\left(t_{0}+t_{1}\right) b_{L}+w_{L}-c_{2} & >t_{0} b_{L}+w_{L}, \\
& \Leftrightarrow \\
\left(t_{0}+t_{1}\right) b_{L}-t_{0} b_{L} & =t_{1} \frac{c_{2}}{t_{1}^{\prime}}>c_{2} .
\end{aligned}
$$


When observing $L$, the agent would not cheat by claiming $H$, if

$$
\left(t_{0}^{\prime}+t_{1}^{\prime}\right) b_{L}-c_{2}+w_{L} \geq\left(t_{0}^{\prime}+t_{1}^{\prime}\right) b_{H}-c_{2}+w_{H}
$$

and

$$
\left(t_{0}^{\prime}+t_{1}^{\prime}\right) b_{L}-c_{2}+w_{L} \geq t_{0}^{\prime} b_{H}+w_{H}
$$

(27) is binding in case that $b_{H} \geq \frac{c_{2}}{t_{1}^{\prime}}$, while (28) is binding in case that $b_{H}<\frac{c_{2}}{t_{1}^{\prime}}$. 